\title{
CARDINAL REPRESENTATIONS FOR CLOSURES AND PRECLOSURES
}

\author{
F. GALVIN, E. C. MILNER, AND M. POUZET
}

\begin{abstract}
A cardinal representation of a preclosure $\varphi$ on a set $E$ is a family $\mathscr{A} \subseteq \mathscr{P}(E)$ such that for any set $X \subseteq \cup \mathscr{A}, \varphi(X)=E$ holds if and only if $|X \cap A|=|A|$ for every $A \in \mathscr{A}$. We show, for example (Theorem 2.3) that any topological closure has such a representation, but there are closures which have no cardinal representation (Theorem 11.2). We prove that, if $k$ is finite and a closure has no independent set of size $k+1$, then it has a cardinal representation, $\mathscr{A}$, of size $|\mathscr{A}| \leq k$ (Theorem 2.4). This result is used to give a new proof of a theorem of D. Duffus and M. Pouzet [4] about gaps in a lattice of finite breadth. We do not know if a closure which has no infinite independent set necessarily has a cardinal representation, but we do prove this is so for the special case of a closure on a countable set (Theorem 2.5). Even in this special case, nothing can be said about the size of the cardinal representation; however, if the closure is algebraic, then there is a finite cardinal representation (Theorem 2.6). These results do not hold for preclosures in general, but if a preclosure on a countable set has no independent set of size $k+1 \quad(k$ finite $)$, then it has a cardinal representation $\mathscr{A}$ of size $|\mathscr{A}| \leq k$ (Theorem 2.7).
\end{abstract}

\section{INTRODUCTION}

We use standard set-theoretic notation. In particular, for a set $X$ and a cardinal $\kappa$, we denote by $[X]^{\kappa}$ (resp. $[X]^{<\kappa},[X]^{\leq \kappa}$ ) the set of all subsets $Y \subseteq X$ having cardinality $|Y|=\kappa$ (resp. $<\kappa, \leq \kappa)$. As usual $\kappa^{+}$denotes the cardinal successor of $\kappa$.

A preclosure on a set $E$ is a function $\varphi: \mathscr{P}(E) \rightarrow \mathscr{P}(E)$ such that (i) $\varphi(\varnothing)=$ $\varnothing$ and (ii) $X \subseteq \varphi(X) \subseteq \varphi(Y)$ whenever $X \subseteq Y \subseteq E$. The subset $X \subseteq E$ is said to be closed with respect to $\varphi$ if $\varphi(X)=X$. A closure on $E$ is a preclosure $\varphi$ which is idempotent, i.e., satisfies (iii) $\varphi(\varphi(X))=\varphi(X)$ for every subset $X \subseteq E$. A topological closure is a closure $\varphi$ which satisfies the remaining Kuratowski axiom (iv) $\varphi(X \cup Y)=\varphi(X) \cup \varphi(Y)$ for all $X, Y \subseteq E$. We also use the terminology of Cohn [2, p. 47] and say that a preclosure $\varphi$ has character

Received by the editors February 2, 1989 and, in revised form, October 2, 1989.

1980 Mathematics Subject Classification (1985 Revision). Primary 06A10, 04A20.

Key words and phrases. Cardinal number, directed graph, quasi-order, algebraic and topological closures, lattice, breadth, independent set.

The first author was partly supported by NSF grants MCS 8102532, DMS 8503732, and DMS 8802856 , and by a visiting professorship at the Université Claude Bernard, Lyon.

The second author was partly supported by NSERC grant \#69.0982 and by a visiting professorship at the Université Claude Bernard, Lyon. 
$n(<\omega)$ if $\varphi(X)=\bigcup\left\{\varphi(Y): Y \in[X]^{\leq n}\right\}$ for every subset $X \subseteq E$; also, we say that $\varphi$ has finite character or is algebraic if $\varphi(X)=\bigcup\left\{\varphi(Y): Y \in[X]^{<\omega}\right\}$ for $X \subseteq E$.

Let $\varphi$ be a preclosure on $E$. A subset $X \subseteq E$ is independent (or $\varphi$ independent $)$ if $x \notin \varphi(X \backslash\{x\})$ for each element $x \in X$; thus, in particular, the empty set, $\varnothing$, is independent. A subset $X \subseteq E$ is dependent if it is not independent. The independence number, $\operatorname{ind}(\varphi)$, of a preclosure $\varphi$ is defined as

$$
\operatorname{ind}(\varphi)=\min \{\kappa:|X|<1+\kappa \text { for every } \varphi \text {-independent set } X \subseteq E\} .
$$

In the case when $\operatorname{ind}(\varphi)=k<\omega$, then $k$ is the size of the largest independent set. A subset $X \subseteq E$ is called a generating set for the preclosure $\varphi$ if $\varphi(X)=E$, and we define the dimension of $\varphi$ to be:

$$
\operatorname{dim}(\varphi)=\min \{|X|: \varphi(X)=E\} .
$$

A preclosure $\varphi$ of character 1 on a set $E$ is determined by its values on singletons and so we can associate with $\varphi$ a directed graph $\mathscr{G}_{\varphi}$ on $E$ in which there is a directed edge $(y, x)$ from $x$ to $y(\neq x)$ if and only if $y \in \varphi(\{x\})$. Conversely, to any (loop-free) directed graph $\mathscr{G}$, there corresponds a preclosure $\varphi$ such that $\mathscr{G}=\mathscr{G}_{\varphi}$. In this case, $\operatorname{ind}(\varphi)$ is the smallest cardinal $\kappa$ such that every subset $X \in[E]^{1+\kappa}$ contains an edge of $\mathscr{G}_{\varphi}$, and $\operatorname{dim}(\varphi)$ is the minimal size of a covering set in $\mathscr{G}_{\varphi}$. In a similar way, corresponding to a closure, $\varphi$, of character 1 , there is a quasi-order (a reflexive, transitive binary relation) $\leq_{\varphi}$ such that $x \leq_{\varphi} y$ if and only if $x \in \varphi(\{y\})$. In this case, the independent subsets are the antichains, and the generating sets are the cofinal subsets, so that $\operatorname{ind}(\varphi)$ is the width of $\left\langle E, \leq_{\varphi}\right\rangle$ and $\operatorname{dim}(\varphi)$ is the cofinality $\operatorname{cf}\left(\left\langle E, \leq_{\varphi}\right\rangle\right)$. (Note also that the closures of character 1 can be characterized as the algebraic topological closures.)

In this paper we are interested in the possibility of representing a family of generating sets of a closure or preclosure in a certain uniform manner. The following definition is suggested by the well-known elementary fact that, for any linearly ordered set $\langle E, \leq\rangle$, there is a well-ordered cofinal subset $A \subseteq E$ such that a set $B \subseteq A$ is cofinal in $\langle E, \leq\rangle$ if, and only if, $|B|=|A|$.

Definition 1.1. A family of sets $\mathscr{A} \subseteq \mathscr{P}(E)$ is called a cardinal representation of the preclosure $\varphi$ on $E$ if, and only if, for any set $X \subseteq \bigcup \mathscr{A}$, we have

$$
\varphi(X)=E \Leftrightarrow|X \cap A|=|A| \quad \text { for every member } A \in \mathscr{A} .
$$

Thus, by the above-mentioned fact, if $\varphi$ is the closure of character 1 corresponding to a linear order, it has a cardinal representation $\mathscr{A}$ of size $|\mathscr{A}|=1$.

There are other uniform ways of generating a family of sets apart from the cardinal representations that we have chosen to consider. For example, call a family $\mathscr{A} \subseteq \mathscr{P}(E)$ a transversal representation for the preclosure $\varphi$ on $E$ if 
$\varphi(\bigcup \mathscr{A})=E$ and, for every set $X \subseteq \bigcup \mathscr{A}$, we have

$$
\varphi(X)=E \Leftrightarrow X \cap A \neq \varnothing \quad \text { for every } A \in \mathscr{A} .
$$

However, this is not quite so interesting since, for any preclosure $\varphi$, the set $\mathscr{A}=\{A \subseteq E: A \cap S \neq \varnothing$ for every generating set $S \subseteq E\}$, is easily seen to be a transversal representation of $\varphi$. For cardinal representations the situation is different. There are closures (in fact there is one of character 3 on a countable set (see Theorem 11.2)) which do not have a cardinal representation, and so it is of interest to know which ones do. Also, apart from giving rise to interesting questions which we have been unable to settle, this notion of cardinal representation may be useful. This is illustrated in $\S 7$, where we use one of our main theorems (Theorem 2.4), to provide a new proof of a result of D. Duffus and M. Pouzet [4] concerning the gaps in a lattice of finite breadth.

\section{Results}

We say that a directed graph or a quasi-order has a cardinal representation if the corresponding preclosure or closure, as described in $\S 1$, has such a representation. Our first result is about countable directed graphs.

Theorem 2.1. A countable directed graph with no infinite independent set has a finite cardinal representation.

It is known that (see [8]) a quasi-ordered set $\langle E, \leq\rangle$ which is either countable or contains no infinite antichain contains a cofinal subset $E^{\prime}$ such that every cofinal subset of $E$ contains a cofinal isomorphic copy of $E^{\prime}$. For general partial orders, however, the cofinal subsets cannot be characterized so simply. Since a cardinal representation of a quasi-ordered set gives some information about cofinal subsets the next result (and also Theorem 2.1) provides some motivation for our initial interest in the notion of cardinal representation. The direct sum of disjoint quasi-orders $\left\langle E_{i}, \leq_{i}\right\rangle(i \in I)$ is a quasi-order $\langle E, \leq\rangle$, where $E=\bigcup\left\{E_{i}: i \in I\right\}$ and $\leq=\bigcup\left\{\leq_{i}: i \in I\right\}$.

Theorem 2.2. (i) Any quasi-ordered set $\langle E, \leq\rangle$ has a cardinal representation, $\mathscr{A}$, of cardinality $|\mathscr{A}| \leq \mathrm{cf}(\langle E, \leq\rangle)$.

(ii) A quasi-ordered set $\langle E, \leq\rangle$ has a finite cardinal representation if and only if there is a cofinal subset of $E$ which is the direct sum of a finite number of chains.

After proving the above theorem about quasi-orders we then realized that, even more generally, every topology has a cardinal representation. By a cardinal representation of a topological space $E$ we mean a cardinal representation for the corresponding closure $\varphi$, where $\varphi(X)$ is the closure of $X$ in this space. A $\pi$-base for the topology is a collection, $\mathscr{B}$, of nonempty open sets such that every nonempty open set contains a member of $\mathscr{B}$. We define $\pi(E)=$ $\min \{|\mathscr{B}|: \mathscr{B}$ is a $\pi$-base $\}$; in other words $\pi(E)=\operatorname{cf}(\langle\mathscr{T}, \supseteq\rangle)$, where $\mathscr{T}$ is the set of nonempty open subsets of $E$. If $\langle E, \leq\rangle$ is a quasi-ordered set, 
then the corresponding closure $\varphi_{\leq}$is topological and so we may regard $E$ as a topological space. Since the set $\left\{U_{x}: x \in A\right\}$ is a $\pi$-base if $A$ is cofinal in $E$ and $U_{x}=\{y \in E: y \geq x\}$, it follows that $\pi(E)=\operatorname{cf}(\langle E, \leq\rangle)$. Thus the following theorem is a generalization of Theorem 2.2(i).

Theorem 2.3. A topological space $E$ has a cardinal representation $\mathscr{A}$ of size $|\mathscr{A}| \leq \pi(E)$.

One of our main results is the next theorem about closures having finitely bounded independent sets. As we have already mentioned, this result will be applied (in $\S 7$ ) to give an alternative proof of a theorem of D. Duffus and M. Pouzet [4] about gaps in a lattice of finite breadth.

Theorem 2.4. Let $\varphi$ be a closure with ind $(\varphi)=k<\omega$. Then $\varphi$ has a cardinal representation, $\mathscr{A}$, such that $|\mathscr{A}| \leq k$ and for each $A \in \mathscr{A},|A|$ is either 1 or a regular infinite cardinal.

We make a few remarks about Theorem 2.4.

Remark 1. From Theorem 2.4 we easily deduce (see Corollary 6.6) a result of E. C. Milner and M. Pouzet [9] which states that: if the dimension, $\operatorname{dim}(\varphi)$, of a closure $\varphi$ is a singular infinite cardinal, then $\operatorname{ind}(\varphi) \geq \omega$.

Remark 2. A closure, $\varphi$, may have cardinal representations of different sizes; also, if $\mathscr{A}$ is a cardinal representation, $A^{\prime} \subseteq A \in \mathscr{A}$ and $\left|A^{\prime}\right|=|A|$, it does not follow that $\varphi\left(A^{\prime}\right)=\varphi(A)$. For example, suppose $\varphi$ is the closure of character 2 defined on $\mathbf{R} \times \mathbf{R}$ by $\varphi\left(\left\{\left(x_{1}, y_{1}\right),\left(x_{2}, y_{2}\right)\right\}\right)=\left\{(u, v): u \leq \max \left\{x_{1}, x_{2}\right\}, v \leq\right.$ $\left.\max \left\{y_{1}, y_{2}\right\}\right\}$. Let $A=\left\{\left(n, \frac{1}{n}\right): 1 \leq n<\omega\right\}, B=\left\{\left(\frac{1}{n}, n\right): 2 \leq n<\omega\right\}, C=$ $\{(n, n): 1 \leq n<\omega\}$. Then $\{A, B\}$ and $\{C\}$ are both cardinal representations for $\varphi$ and $\varphi(A \backslash\{(1,1)\}) \neq \varphi(A)$.

Remark 3. The finiteness of ind $(\varphi)$ is an essential condition for Theorem 2.4. Like Dilworth's theorem [3] about the decomposition of a partially ordered set into finitely many chains, this theorem fails badly when $\operatorname{ind}(\varphi) \geq \omega$. For example, consider the closure $\varphi$ of character 1 defined on $\kappa \times \kappa^{+}$, where $\kappa$ is any regular infinite cardinal, by $\varphi(\{(\alpha, \beta)\})=\left\{\left(\alpha^{\prime}, \beta^{\prime}\right): \alpha^{\prime} \leq \alpha\right.$ and $\left.\beta^{\prime} \leq \beta\right\}$. In this case, there is no infinite independent set, $\operatorname{ind}(\varphi)=\omega$, but it can be shown that there is no cardinal representation, $\mathscr{A}$, of cardinality $|\mathscr{A}|<\kappa$ (in fact, there is no weak cardinal representation (see Definition 2.1) of size less than $\kappa$, although there is a cardinal representation of size $\kappa$ ).

This last example shows that, if a closure $\varphi$ has independence number $\operatorname{ind}(\varphi)=\omega$, then no bound can be placed upon the size of a cardinal representation for $\omega$. We do not know if such a closure necessarily has a cardinal representation.

Problem 1. Is there a closure $\varphi$ with $\operatorname{ind}(\varphi)=\omega$ which has no cardinal representation?

We can answer the above question for closures on a countable set. 
Theorem 2.5. If $\varphi$ is a closure on $\omega$ such that $\operatorname{ind}(\varphi) \leq \omega$, then $\varphi$ has a cardinal representation.

Nothing can be said about the size of the cardinal representation whose existence is guaranteed by Theorem 2.5. We give an example (Theorem 11.1) of a $T_{1}$-topological closure, $\varphi$, on $\omega$ such that $\operatorname{ind}(\varphi)=\omega$ and for which there is no countable cardinal representation, and it is consistent that there is no cardinal representation of size less than $2^{\aleph_{0}}$ (and $2^{\aleph_{0}}$ is arbitrarily large). The condition that $\operatorname{ind}(\varphi) \leq \omega$ is needed in Theorem 2.5. We give an example (Theorem 11.2) of an algebraic closure of character 3 on $\omega$ which has no cardinal representation at all (but there are infinite independent sets). Since, by Theorem 2.2(i), a closure of character 1 always has a cardinal representation, this leaves open a question about character 2 closures.

Problem 2. Is there a closure of character 2 which has no cardinal representation?

For algebraic closures, Theorem 2.5 can be improved.

Theorem 2.6. If $\varphi$ is an algebraic closure on $\omega$ and $\operatorname{ind}(\varphi) \leq \omega$, then $\varphi$ has a finite cardinal representation.

It is convenient to consider the following weakening of Definition 1.1.

Definition 2.1. If $\varphi$ is a preclosure on $E$, then a family $\mathscr{A} \subseteq \mathscr{P}(E)$ is called a weak cardinal representation of $\varphi$ if, whenever $X \subseteq \cup \mathscr{A}$ is such that $|X \cap A|=$ $|A|$ for every member $A \in \mathscr{A}$, then $X$ is a generating set, i.e. $\varphi(X)=E$.

Of course, the set of singletons, $[S]^{1}$, is trivially a weak cardinal representation of $\varphi$ if $S$ is a generating set. Consequently, a question of interest is whether a closure of preclosure, $\varphi$, has a weak cardinal representation, $\mathscr{A}$, of size $|\mathscr{A}|<\operatorname{dim}(\varphi)$. It is natural to ask if our Theorems $2.4,2.5$, and 2.6 about closures have analogues for preclosures (possibly with the weaker conclusion that there is a (nontrivial) weak cardinal representation).

Theorem 2.4 fails badly for preclosures. Z. Nagy and Z. Szentmiklóssy [10] have shown that, for any cardinal $\kappa \geq \omega$, there is a tournament (i.e., a complete graph in which each edge is given an orientation) on a set of size $\left(\kappa^{<\kappa}\right)^{+}$for which there is no weak cardinal representation, $\mathscr{A}$, of cardinality $|\mathscr{A}|<\kappa$. (We originally asked if there is a tournament which has no finite weak cardinal representation, and $\mathrm{A}$. Hajnal gave an example which depended upon $\mathrm{CH}$; the construction in [10] does not use any additional set-theoretic hypothesis.) Of course, a tournament corresponds to a preclosure of character 1 and independence number 1 . However, there is an analogue of Theorem 2.4 for preclosures on a countable set.

Theorem 2.7. Let $\varphi$ be a preclosure on $\omega$ such that $\operatorname{ind}(\varphi)=k<\omega$. Then $\varphi$ has a cardinal representation, $\mathscr{A}$, with $|\mathscr{A}| \leq k$.

We do not know if there are analogues of Theorems 2.5 and 2.6 for preclosures. 
Problem 3. Is there a preclosure, $\varphi$, on $\omega$ such that $\operatorname{ind}(\varphi)=\omega$ and $\varphi$ has no cardinal representation?

Problem 4. Is there an algebraic preclosure, $\varphi$, on $\omega$ such that $\operatorname{ind}(\varphi)=\omega$ and $\varphi$ has no (or no finite) cardinal representation?

Of course, by Theorem 2.1, we do know the answer to Problem 4 in the case of preclosures of character 1.

We first proved Theorem 2.7 with the seemingly weaker conclusion that: $\varphi$ has a weak cardinal representation, $\mathscr{A}$, of size $|\mathscr{A}| \leq k$. The stronger conclusion follows from this and the following slightly surprising result.

Theorem 2.8. Suppose that $\mathscr{A}=\left\{A_{1}, \ldots, A_{k}\right\}$ is a finite weak cardinal representation of a preclosure $\varphi$ such that $\bigcup \mathscr{A}$ is countable. Then $\varphi$ has a cardinal representation $\mathscr{A}^{\prime}=\left\{A_{1}^{\prime}, \ldots, A_{k}^{\prime}\right\}$ such that $\bigcup \mathscr{A}^{\prime} \subseteq \bigcup \mathscr{A}$ and $\left|A_{i}^{\prime}\right| \leq\left|A_{i}\right|$ $(1 \leq i \leq k)$.

Corollary 2.9. A preclosure on $\omega$ has a finite cardinal representation if and only if it has a finite weak cardinal representation.

The condition in Theorem 2.8 that the members of $\mathscr{A}$ be countable sets is essential. For example, consider the (topological) closure $\varphi$ defined on the real line $\mathbf{R}$ by setting $\varphi(X)=\mathbf{R}$ if $X$ has uncountable closure in the usual topology on $\mathbf{R}$ (equivalently, if $X$ contains a subset order isomorphic to the set $\mathbf{Q}$ of all rational numbers), and $\varphi(X)=X$ otherwise. Clearly $\{A\}$ is a weak cardinal representation of size 1 , where $A$ is any uncountable set of real numbers. On the other hand, it is easy to see that every cardinal representation of $\varphi$ has cardinality $2^{\aleph_{0}}$. (Note that $\varphi$ does have cardinal representations, e.g. $\mathscr{A}=\{\mathbf{Q} \backslash X: \varphi(X) \neq \mathbf{R}\}$.)

In general, the members of a cardinal representation or weak cardinal representation of a preclosure are not disjoint from one another. However, in the case when a preclosure has a finite (weak) cardinal representation, then it can be shown that there is also such a representation in which the members are pairwise disjoint.

Theorem 2.10. Suppose that $\mathscr{A}$ is a finite (weak) cardinal representation of a preclosure. Then there are $\mathscr{A}^{\prime}$ and an injective map $f: \mathscr{A}^{\prime} \rightarrow \mathscr{A}$ such that (i) $\mathscr{A}^{\prime}$ is a (weak) cardinal representation whose members are pairwise disjoint, and (ii) $A^{\prime} \subseteq f\left(A^{\prime}\right)$ for each $A^{\prime} \in \mathscr{A}^{\prime}$, and $\left|A^{\prime}\right|=\left|f\left(A^{\prime}\right)\right|$ whenever $\left|f\left(A^{\prime}\right)\right|$ is infinite.

3. Finite Cardinal Representations; proofs of Theorems 2.8 and 2.10

Proof of Theorem 2.8. Let $k<\omega$ and let $\mathscr{A}=\left\{A_{1}, \ldots, A_{k}\right\}$ be a finite weak cardinal representation of a preclosure $\varphi$ on $E$ with $|\bigcup \mathscr{A}| \leq \omega$. With $k$ fixed, let $W$ be the set of all sequences $\left(B_{1}, \ldots, B_{k}\right)$ such that $\left\{B_{1}, \ldots, B_{k}\right\}$ is a weak cardinal representation of $\varphi$. The set $W$ is quasi-ordered by the rule: $\left(B_{1}^{\prime}, \ldots, B_{k}^{\prime}\right) \leq\left(B_{1}, \ldots, B_{k}\right)$ if and only if $B_{1}^{\prime} \cup \cdots \cup B_{k}^{\prime} \subseteq B_{1} \cup \cdots \cup$ 
$B_{k}$ and $\left|B_{i}^{\prime}\right| \leq\left|B_{i}\right|$ for $1 \leq i \leq k$. For $\left(B_{1}, \ldots, B_{k}\right) \in W$ we define $W\left(B_{1}, \ldots, B_{k}\right)=\left\{\left(B_{1}^{\prime}, \ldots, B_{k}^{\prime}\right) \in W:\left(B_{1}^{\prime}, \ldots, B_{k}^{\prime}\right) \leq\left(B_{1}, \ldots, B_{k}\right)\right\}$. We are given $\left(A_{1}, \ldots, A_{k}\right) \in W$ such that $\left|A_{1} \cup \cdots \cup A_{k}\right| \leq \omega$; we have to show that, for some $\left(A_{1}^{\prime}, \ldots, A_{k}^{\prime}\right) \in W\left(A_{1}, \ldots, A_{k}\right),\left\{A_{1}^{\prime}, \ldots, A_{k}^{\prime}\right\}$ is a cardinal representation of $\varphi$. Without loss of generality we may assume that

(3.1) there is no $\left(A_{1}^{\prime}, \ldots, A_{k}^{\prime}\right) \in W\left(A_{1}, \ldots, A_{k}\right)$ with $\left|A_{i}^{\prime}\right|<\left|A_{i}\right|$ for some $i$.

We will say that $B_{i}$ is inessential in $\left(B_{1}, \ldots, B_{k}\right)$ if there is a set $X \subseteq B_{i}$ such that $|X|<\left|B_{i}\right|$ and $\varphi\left(X \cup \bigcup\left\{B_{j}: j \neq i\right\}\right)=E$. Let iness $\left(B_{1}, \ldots, B_{k}\right)=$ $\mid\left\{i: B_{i}\right.$ is inessential in $\left.\left(B_{1}, \ldots, B_{k}\right)\right\} \mid$. Clearly, if $\left(B_{1}, \ldots, B_{k}\right) \in W$ and iness $\left(B_{1}, \ldots, B_{k}\right)=0$, then $\left\{B_{1}, \ldots, B_{k}\right\}$ is a cardinal representation of $\varphi$. Ne may assume

$$
\begin{aligned}
& \text { there is no }\left(A_{1}^{\prime}, \ldots, A_{k}^{\prime}\right) \in W\left(A_{1}, \ldots, A_{k}\right) \text { with } \\
& \quad \operatorname{iness}\left(A_{1}^{\prime}, \ldots, A_{k}^{\prime}\right)<\operatorname{iness}\left(A_{1}, \ldots, A_{k}\right) .
\end{aligned}
$$

In particular, if $\left(A_{1}^{\prime}, \ldots, A_{k}^{\prime}\right) \in W$ and $A_{i}^{\prime} \subseteq A_{i}$ for $1 \leq i \leq k$, and if $A_{j}$ is inessential in $\left(A_{1}, \ldots, A_{k}\right)$, then $A_{j}^{\prime}$ is inessential in $\left(A_{1}^{\prime}, \ldots, A_{k}^{\prime}\right)$.

We will finish the proof by showing that iness $\left(A_{1}, \ldots, A_{k}\right)=0$. Assume the contrary; without loss of generality, we may suppose that $A_{1}$ is inessential in $\left(A_{1}, \ldots, A_{k}\right)$. We may also assume that $A_{2}, \ldots, A_{r}$ are (countably) infinite and that $A_{r+1}, \ldots, A_{k}$ are finite. Let $F=A_{r+1} \cup \cdots \cup A_{k}$.

Case 1. $A_{1}$ is finite. Given any $x \in A_{1}$ and any infinite sets $B_{i} \subseteq A_{i} \quad(2 \leq i \leq$ $r)$, there must be infinite sets $B_{i}^{\prime} \subseteq B_{i}(2 \leq i \leq r)$ such that

$$
\varphi\left(\left(A_{1} \backslash\{x\}\right) \cup B_{2}^{\prime} \cup \cdots \cup B_{r}^{\prime} \cup F\right) \neq E ;
$$

otherwise we would have

$$
\left(A_{1} \backslash\{x\}, B_{2}, \ldots, B_{r}, A_{r+1}, \ldots, A_{k}\right) \in W\left(A_{1}, \ldots, A_{k}\right)
$$

and $\left|A_{1} \backslash\{x\}\right|<\left|A_{1}\right|$, contradicting (3.1). Hence there are infinite sets $C_{i} \subseteq A_{i}$ $(2 \leq i \leq r)$ such that $\varphi\left(\left(A_{1} \backslash\{x\}\right) \cup C_{2} \cup \cdots \cup C_{r} \cup F\right) \neq E$ for every $x \in A_{1}$. But then $A_{1}$ is not inessential in $\left(A_{1}, C_{2}, \ldots, C_{r}, A_{r+1}, \ldots, A_{k}\right)$, contradicting (3.2).

Case 2. $A_{1}$ is infinite. Let $A_{1}=\left\{x_{1}^{n}: n<\omega\right\}$. For any finite set $X \subseteq$ $A_{1} \cup \cdots \cup A_{k}$ and any infinite sets $B_{i} \subseteq A_{i}(2 \leq i \leq r)$ there must be infinite sets $B_{i}^{\prime} \subseteq B_{i} \quad(2 \leq i \leq r)$, such that $\varphi\left(X \cup B_{2}^{\prime} \cup \cdots \cup B_{r}^{\prime} \cup F\right) \neq E$; otherwise, we would have $\left(X, B_{2}, \ldots, B_{r}, A_{r+1}, \ldots, A_{k}\right) \in W\left(A_{1}, \ldots, A_{k}\right)$ and $|X|<$ $\left|A_{1}\right|$, contradicting (3.1). Choose elements $x_{i}^{0} \in A_{i}(2 \leq i \leq r)$ and let $X^{0}=$ $\left\{x_{i}^{0}: 1 \leq i \leq r\right\}$. By the preceding remark, we can choose infinite sets $A_{i}^{0} \subseteq A_{i}$ $(2 \leq i \leq r)$ so that $\varphi\left(X^{0} \cup A_{2}^{0} \cup \cdots \cup A_{r}^{0} \cup F\right) \neq E$. Now let $0<n<\omega$ and suppose that, for $2 \leq i \leq r$, elements $x_{i}^{0}, \ldots, x_{i}^{n-1} \in A_{i}$ and infinite sets $A_{i}^{n-1} \subseteq \cdots \subseteq A_{i}^{0} \subseteq A_{i}$ have already been chosen. Choose elements $x_{i}^{n} \in$ 
$A_{i}^{n-1} \backslash\left\{x_{i}^{0}, \ldots, x_{i}^{n-1}\right\} \quad(2 \leq i \leq r)$ and let $X^{n}=\left\{x_{i}^{j}: 0 \leq j \leq n, 1 \leq i \leq r\right\}$. Then choose infinite sets $A_{i}^{n} \subseteq A_{i}^{n-1}(2 \leq i \leq r)$ so that

$$
\varphi\left(X^{n} \cup A_{2}^{n} \cup \cdots \cup A_{r}^{n} \cup F\right) \neq E .
$$

Finally, let $C_{i}=\left\{x_{i}^{n}: n<\omega\right\} \quad(2 \leq i \leq r)$. Then $C_{i}$ is an infinite subset of $A_{i}$, and so we have $\left(A_{1}, C_{2}, \ldots, C_{r}, A_{r+1}, \ldots, A_{k}\right) \in W\left(A_{1}, \ldots, A_{k}\right)$. By the construction, for each $n<\omega$ we have $\left\{x_{1}^{0}, \ldots, x_{1}^{n}\right\} \cup C_{2} \cup \cdots \cup C_{r} \subseteq$ $X^{n} \cup A_{2}^{n} \cup \cdots \cup A_{r}^{n}$, and so $\varphi\left(\left\{x_{1}^{0}, \ldots, x_{1}^{n}\right\} \cup C_{2} \cup \cdots \cup C_{r} \cup F\right) \neq E$. But then $A_{1}$ is not inessential in $\left(A_{1}, C_{2}, \ldots, C_{r}, A_{r+1}, \ldots, A_{k}\right)$, contradicting (3.2).

Proof of Theorem 2.10. Let $\mathscr{A}$ be a finite (weak) cardinal representation of a preclosure and let $\mathscr{A}_{0}=\{A \in \mathscr{A}: A$ finite $\}, \mathscr{A}_{1}=\mathscr{A} \backslash \mathscr{A}_{0}$. We may assume that any member of $\mathscr{A}_{0}$ is disjoint from any other member of $\mathscr{A}$. Let $\mathscr{B}$ be a finite family of pairwise disjoint subsets of $\bigcup \mathscr{A}_{1}$ such that $A=\bigcup\{B \in \mathscr{B}: B \subseteq A\}$ for each $A \in \mathscr{A}_{1}$ (e.g. let $\mathscr{B}$ be the set of all atoms in the subalgebra generated by $\mathscr{A}_{1}$ in the Boolean algebra of all subsets of $\left.\cup \mathscr{A}_{1}\right)$. Choose a minimal family $\mathscr{B}_{1} \subseteq \mathscr{B}$ such that

$$
\left(\forall A \in \mathscr{A}_{1}\right)\left(\exists B \in \mathscr{B}_{1}\right) \quad(B \subseteq A \text { and }|B|=|A|) .
$$

Then, for each $A^{\prime} \in \mathscr{A}^{\prime}=\mathscr{A}_{0} \cup \mathscr{B}_{1}$, there is $A=f\left(A^{\prime}\right) \in \mathscr{A}$ such that $A^{\prime} \subseteq A$ and $\left|A^{\prime}\right|=|A|$ and $f\left(A^{\prime}\right) \neq f\left(A^{\prime \prime}\right)$ if $A^{\prime} \neq A^{\prime \prime}$. The members of $\mathscr{A}^{\prime}$ are pairwise disjoint, and we have only to show that it is a (weak) cardinal representation.

Consider any set $X \subseteq \cup \mathscr{A}^{\prime} \subseteq \cup \mathscr{A}$. If $\left|X \cap A^{\prime}\right|=\left|A^{\prime}\right|$ for every set $A^{\prime} \in \mathscr{A}^{\prime}$, then, by (3.3), $|X \cap A|=|A|$ for every $A \in \mathscr{A}$, and hence $X$ is a generating set. Thus $\mathscr{A}^{\prime}$ is a weak cardinal representation. Now suppose that $\mathscr{A}$ is a cardinal representation and that $X \subseteq \cup \mathscr{A}^{\prime}$ is a generating set. Then $|X \cap A|=|A|$ for each $A \in \mathscr{A}$ and it follows that (3.3) holds with $\mathscr{B}_{1}$ replaced by $\mathscr{B}_{2}^{\prime}=\{B \in$ $\left.\mathscr{B}_{1}:|X \cap B|=|B|\right\}$. By the minimality of $\mathscr{B}_{1}$ it follows that $\mathscr{B}_{2}^{\prime}=\mathscr{B}_{1}$, and hence that $\mathscr{A}^{\prime}$ is also a cardinal representation.

\section{Countable directed graphs. Proof of Theorem 2.1}

In this section we prove Theorem 2.1 that a countable directed graph with no infinite independent set has a finite cardinal representation. Our original proof of this result was rather more complicated than the one presented here which uses a special case (Lemma 4.1) of a theorem of S. Todorčević [11].

Let $\mathscr{G}=(E, \mathscr{E})$ be a directed graph with edge set $\mathscr{E} \subseteq E \times E$. For $X \subseteq E$, we define $\mathscr{G}(X)=X \cup\{y \in E:(y, x) \in \mathscr{E}$ for some $x \in X\}$. We say that a set $X \subseteq E$ is finitely compatible if, for every finite set $F \subseteq X$, there is an element $x \in E$ such that $F \subseteq \mathscr{G}(\{x\})$; a set $X \subseteq E$ is directed if it satisfies the stronger condition that, for every finite set $F \subseteq X$, there is $x \in X$ such that $F \subseteq \mathscr{G}(\{x\})$. 
Lemma 4.1. If the directed graph $\mathscr{G}=(E, \mathscr{E})$ has no infinite independent set, then $E$ is a finite union of directed sets.

Proof. Suppose for a contradiction that $E=E_{0}$ is not a finite union of directed sets. Let $n<\omega$ and suppose that we have already chosen $x_{i} \in E \quad(i<n)$ and $E_{n} \subseteq E$ so that $E_{n}$ is not a finite union of directed sets. Then there is a finite set $X \subseteq E_{n}$ such that $X \nsubseteq \mathscr{G}(\{y\})$ holds for every $y \in E_{n}$. Hence $E_{n}=\bigcup\left\{E_{n, x}: x \in X\right\}$, where $E_{n, x}=\left\{y \in E_{n}: x \notin \mathscr{G}(\{y\})\right\}$. If for each $x \in X$ the set $E_{n, x} \backslash \mathscr{G}(\{x\})$ is a finite union of directed sets, then so also is $E_{n}$. Therefore, for some $x=x_{n} \in X, E_{n+1}=E_{n, x} \backslash \mathscr{G}(\{x\})$ is not a finite union of directed sets. This defines $x_{n}(n<\omega)$ so that $\left\{x_{n}: n<\omega\right\}$ is an infinite independent set.

Theorem 2.1 is an immediate consequence of Theorem 2.8, Lemma 4.1, and the following fact.

Lemma 4.2. For a countable directed graph $\mathscr{G}=(E, \mathscr{E})$ and a positive integer $n$, the following statements are equivalent:

(i) $E$ is a union of $n$ finitely compatible sets;

(ii) $\mathscr{G}$ has a weak cardinal representation $\mathscr{A}$ such that $|\mathscr{A}| \leq n$ and each finite member of $\mathscr{A}$ is a singleton.

Proof. Suppose that (i) holds and $\omega=E=\bigcup\left\{E_{i}: i<n\right\}$, where each $E_{i}$ $(i<n)$ is finitely compatible. Then there are elements $x_{i, k} \in E \quad(i<n ; k \in \omega)$ such that $E_{i} \cap\{0,1, \ldots, k\} \subseteq \mathscr{G}\left(\left\{x_{i, k}\right\}\right)$. Put $A_{i}=\left\{x_{i, k}: k \in \omega\right\} \quad(i<n)$. If $A_{i}$ is finite, then $x_{i, k}=x_{i}$ for infinitely many $k$ and so $E_{i} \subseteq \mathscr{G}\left(\left\{x_{i}\right\}\right)$; in this case we may assume that $A_{i}=\left\{x_{i}\right\}$. If $A_{i}$ is infinite, then $E_{i} \subseteq \mathscr{G}\left(A_{i}^{\prime}\right)$ for any infinite set $A_{i}^{\prime} \subseteq A_{i}$. It follows that $\mathscr{A}=\left\{A_{i}: i<n\right\}$ is a weak cardinal representation satisfying (ii).

Next, assume that (ii) holds. For $A \in \mathscr{A}$ let $E_{A}=\bigcap\left\{\mathscr{G}\left(A^{\prime}\right): A^{\prime} \subseteq A\right.$ and $\left.\left|A^{\prime}\right|=|A|\right\}$. We claim that $E=\bigcup\left\{E_{A}: A \in \mathscr{A}\right\}$ and that $E_{A}(A \in \mathscr{A})$ is finitely compatible. Suppose for a contradiction that there is an element $x \in E \backslash \bigcup\left\{E_{A}: A \in \mathscr{A}\right\}$. Then, for each $A \in \mathscr{A}$, there is $A^{\prime} \subseteq A$ such that $\left|A^{\prime}\right|=|A|$ and $x \notin \mathscr{G}\left(A^{\prime}\right)$. But this is a contradiction since

$$
E=\mathscr{G}\left(\bigcup\left\{A^{\prime}: A \in \mathscr{A}\right\}\right)=\bigcup\left\{\mathscr{G}\left(A^{\prime}\right): A \in \mathscr{A}\right\} .
$$

Thus $E$ is the union of the sets $E_{A}(A \in \mathscr{A})$. Now suppose that there is $A \in \mathscr{A}$ such that $E_{A}$ is not finitely compatible. Then $A$ is infinite and there is a finite set $F \subseteq E_{A}$ such that $F \nsubseteq \mathscr{G}(\{x\})$ for every $x \in E$. It follows that there is some $p \in F$ such that $A^{\prime}=\{x \in A: p \notin \mathscr{G}(\{x\})\}$ has the same cardinality as $A$. This is a contradiction since $p \in F \subseteq E_{A} \subseteq \mathscr{G}\left(A^{\prime}\right)$.

From Lemma 4.2 and Theorem 2.8 we obtain the following corollaries.

Corollary 4.3. Let $\mathscr{G}=(E, \mathscr{E})$ be a countable directed graph, and let $n<\omega$. If $E$ is the union of $n$ finitely compatible sets, then $\mathscr{G}$ has a cardinal representation $\mathscr{A}$ of size $|\mathscr{A}| \leq n$. 
Corollary 4.4. A countable directed graph $\mathscr{G}=(E, \mathscr{E})$ has a finite cardinal representation if and only if $E$ is a finite union of finitely compatible sets.

We can say a little more concerning these corollaries. Let $\mathscr{G}=(E, \mathscr{E})$ be a countable directed graph, and let $n<\omega$. Consider the following statements:

(a) $\mathscr{G}$ has a cardinal representation $\mathscr{A}$ such that $|\mathscr{A}| \leq n$ and each finite member of $\mathscr{A}$ is a singleton.

(b) $\mathscr{G}$ has a weak cardinal representation $\mathscr{A}$ such that $|\mathscr{A}| \leq n$ and each finite member of $\mathscr{A}$ is a singleton.

(b') $E$ is the union of $n$ finitely compatible sets.

(c) $\mathscr{G}$ has a weak cardinal representation $\mathscr{A}$ such that $|\mathscr{A}| \leq n$.

(c') $\mathscr{G}$ has a cardinal representation $\mathscr{A}$ such that $|\mathscr{A}| \leq n$.

Then the following implications hold:

$$
(\mathrm{a}) \Rightarrow(\mathrm{b}) \Leftrightarrow\left(\mathrm{b}^{\prime}\right) \Rightarrow(\mathrm{c}) \Leftrightarrow\left(\mathrm{c}^{\prime}\right) \text {. }
$$

The implications $(\mathrm{a}) \Rightarrow(\mathrm{b}) \Rightarrow$ (c) are trivial; (b) $\Leftrightarrow\left(\mathrm{b}^{\prime}\right)$ by Lemma 4.2; and $(\mathrm{c}) \Leftrightarrow\left(\mathrm{c}^{\prime}\right)$ by Theorem 2.8. However, for $n>0$ there are no other implications. To see that (c) does not imply (b), consider the graph $\mathscr{G}$ when $|E|=n+1$ and $\mathscr{E}=\varnothing$. To see that (b) does not imply (a), choose a surjection $f: \omega \backslash\{0,1, \ldots, n-1\} \rightarrow[\omega]^{n}$ such that $f(y) \subseteq\{0,1, \ldots, y-1\}$ and consider the graph $\mathscr{G}=(E, \mathscr{E})$ with $E=\omega$ and $\mathscr{E}=\{(y, x): y<x$ or $x \notin f(y)\}$. Clearly, for $X \subseteq \omega$, one has $\mathscr{G}(X)=E \Leftrightarrow|X|>n .\{\omega\}$ is a weak cardinal representation for $\mathscr{G}$ (and $\omega$ is a directed set), but $\mathscr{G}$ has no cardinal representation of size $\leq n$ such that each finite member is a singleton. In fact, ä family $\mathscr{A} \subseteq \mathscr{P}(E)$ is a cardinal representation if and only if $|\bigcup \mathscr{A}|=n+1$.

\section{Quasi-orders AND topologies. Proofs of Theorems 2.2 AND 2.3}

We already observed in $\S 2$ that part (i) of Theorem 2.2 is a special case of Theorem 2.3, and so we begin this section with a proof of this result about topological closures.

For the convenience of the reader we repeat here the definitions already stated in $\S 2$. A $\pi$-base of a topological space $E$ is a set, $\mathscr{B}$, of nonempty open subsets of $E$ such that every nonempty open set contains a member of $\mathscr{B}$, and $\pi(E)=\min \{|\mathscr{B}|: \mathscr{B}$ is a $\pi$-base of $E\}$.

The density of the space $E$ is $d(E)=\min \{|X|: X$ is dense in $E\}$. A subspace $E^{\prime} \subseteq E$ is uniformly dense if $d\left(E^{\prime \prime}\right)=\left|E^{\prime}\right|$ for every nonempty (relatively) open subset $E^{\prime \prime} \subseteq E^{\prime}$. We say that $E$ is the direct sum of subspaces $E_{i}(i \in I)$ if the $E_{i}(i \in I)$ are pairwise disjoint open subsets with union $E$.

Lemma 5.1. A topological space $E$ contains a dense subset which is a direct sum of uniformly dense subspaces.

Proof. Let $\mathscr{B}$ denote the set of all nonempty open subsets $B \subseteq E$ with the property that every nonempty open subset of $B$ has the same density as $B$. Let $\mathscr{B}_{1}$ be a maximal subfamily of pairwise disjoint members of $\mathscr{B}$. Then $\mathscr{B}$ 
is a $\pi$-base and $\bigcup \mathscr{B}_{1}$ is dense in $E$. For each $B \in \mathscr{B}_{1}$ choose a dense subset $Y_{B}$ of cardinality $\left|Y_{B}\right|=d(B)$. Then $Y=\bigcup\left\{Y_{B}: B \in \mathscr{B}_{1}\right\}$ is dense in $E$ and is the direct sum of the $Y_{B}$, and each $Y_{B}$ is uniformly dense.

Proof of Theorem 2.3. Let $E$ be a topological space. By Lemma 5.1 there is a dense subset $Y$ which is a direct sum of uniformly dense subspaces $Y_{i}$ $(i \in I)$. For each $i$, let $\mathscr{A}_{i}$ be a $\pi$-base for $Y_{i}$ of size $\pi\left(Y_{i}\right)$. We claim that $\mathscr{A}=\bigcup\left\{\mathscr{A}_{i}: i \in I\right\}$ is a cardinal representation. Let $X \subseteq \bigcup \mathscr{A} \subseteq Y$. If $X$ is dense in $E$, then, for each $i, X$ is dense in $Y_{i}$ and hence $|X \cap A|=\left|Y_{i}\right|=|A|$ for each $A \in \mathscr{A}_{i}$. On the other hand, if $|X \cap A|=|A| \quad(\neq 0)$ for each $A \in \mathscr{A}$, then $X$ is dense in each $Y_{i}$ and so $X$ is dense in $E$. Thus $\mathscr{A}$ is a cardinal Fepresentation and $|\mathscr{A}|=\sum\left\{\left|\mathscr{A}_{i}\right|: i \in I\right\}=\sum\left\{\pi\left(Y_{i}\right): i \in I\right\}=\pi(Y) \leq \pi(E)$, since $Y$ is dense in $E$.

We shall now prove part (ii) of Theorem 2.2. Let $\langle E, \leq\rangle$ be a quasi-ordered set. The quasi-order induced on a subset $E^{\prime} \subseteq E$ will be denoted by $\left\langle E^{\prime}, \leq\right\rangle$ instead of the more formally correct $\left\langle E^{\prime},\left(E^{\prime} \times E^{\prime}\right) \cap \leq\right\rangle$. For $A \subseteq E$ and $x \in E$, we define $A(\leq x)=\{a \in A: a \leq x\}, A(\geq x)=\{a \in A: a \geq x\}$; also we define $\widehat{A}=\bigcup\{E(\leq a): a \in A\}$.

Part (ii) of Theorem 2.2 is the equivalence (a) $\Leftrightarrow$ (d) of the following result.

Theorem 5.2. For any quasi-ordered set $\langle E, \leq\rangle$, the following statements are equivalent:

(a) $\langle E, \leq\rangle$ has a finite cardinal representation;

(b) $\langle E, \leq\rangle$ has a finite weak cardinal representation;

(c) E contains a cofinal subset which is a union of a finite number of chains (i.e. a cofinal subset of finite width);

(d) E contains a cofinal subset which is the direct sum of a finite number of chains.

Proof. (a) $\Rightarrow$ (b) Obvious.

(c) $\Rightarrow$ (d) Let $C_{1}, \ldots, C_{n}$ be chains whose union is cofinal in $E$; we may assume that $n$ is as small as possible. By the minimality of $n$ it follows that, for each $i \quad(1 \leq i \leq n)$, there is $x_{i} \in C_{i} \backslash \bigcup\left\{\widehat{C}_{j}: j \neq i\right\}$. Let $C_{i}^{\prime}=C_{i}\left(\geq x_{i}\right)$. Then the set $E^{\prime}=C_{1}^{\prime} \cup \cdots \cup C_{n}^{\prime}$ is cofinal in $E$ and is a direct sum of the chains $C_{i}^{\prime}(1 \leq i \leq n)$.

(d) $\Rightarrow$ (a) Suppose that $E$ contains a cofinal subset which is a direct sum of chains $C_{i}(i \in I)$. Let $A_{i}$ be a cofinal subset of the chain $\left\langle C_{i}, \leq\right\rangle$ of order type $\operatorname{cf}\left(\left\langle C_{i}, \leq\right\rangle\right)(i \in I)$. Then $\mathscr{A}=\left\{A_{i}: i \in I\right\}$ is a cardinal representation of $\langle E, \leq\rangle$ which is finite if $I$ is finite.

(b) $\Rightarrow$ (c) This is an immediate consequence of the following two lemmas.

Lemma 5.3. If the quasi-ordered set $\langle E, \leq\rangle$ has a finite weak cardinal representation, then it has a finite weak cardinal representation $\mathscr{A}$ such that, for each $A \in \mathscr{A}$, every subset $A^{\prime} \subseteq A$ having the same cardinality as $A$ is cofinal in $A$. Proof. Let $\left\{A_{1}, \ldots, A_{n}\right\}$ be a finite weak cardinal representation for $\langle E, \leq\rangle$. 
We may assume that $n$ is minimal. For $1 \leq i \leq n$, let $B_{i}=\bigcap\left\{\widehat{X}: X \subseteq A_{i}\right.$, $\left.|X|=\left|A_{i}\right|\right\}$.

Suppose that there is an element $x \in E \backslash\left(B_{1} \cup \cdots \cup B_{n}\right)$. Then, for each $i$ $(1 \leq i \leq n)$, there is a set $X_{i} \subseteq A_{i}$ such that $\left|X_{i}\right|=\left|A_{i}\right|$ and $x \notin \widehat{X}_{i}$. Then $x \notin \widehat{X}$, where $X=X_{1} \cup \cdots \cup X_{n}$, and this is a contradiction since $\left|X \cap A_{i}\right|=\left|A_{i}\right|$ $(1 \leq i \leq n)$ and $\left\{A_{1}, \ldots, A_{n}\right\}$ is a weak cardinal representation for $\langle E, \leq\rangle$. Hence $B_{1} \cup \cdots \cup B_{n}=E$.

We claim that

$$
\left|A_{i} \cap B_{j}\right|<\left|A_{i}\right| \text { for } i \neq j
$$

Indeed, suppose that $i \neq j$ and that $\left|A_{i} \cap B_{j}\right|=\left|A_{i}\right|$. We obtain a contradiction by showing that $\left\{A_{k}: 1 \leq k \leq n, k \neq i\right\}$ is a weak cardinal representation. Let $X \subseteq E$ and suppose that $\left|X \cap A_{k}\right|=\left|A_{k}\right|$ for $1 \leq k \leq n$ and $k \neq i$. Then $\widehat{Y} \supseteq B_{j}$, where $Y=X \cap A_{j}$, and so $\left|\widehat{X} \cap A_{i}\right| \geq\left|\widehat{Y} \cap A_{i}\right| \geq\left|B_{j} \cap A_{i}\right|=\left|A_{i}\right|$. Therefore $\widehat{X}$ is cofinal in $\langle E, \leq\rangle$ and hence so also is $X$. This proves (5.1).

We claim also that

$$
\left|A_{i} \cap B_{i}\right|=\left|A_{i}\right| \text { for } 1 \leq i \leq n .
$$

If $A_{i}$ is infinite this follows from (5.1) since $B_{1} \cup \cdots \cup B_{n}=E$; if $A_{i}$ is finite, then $B_{i}=\widehat{A_{i}} \supseteq A_{i}$ and hence $\left|A_{i} \cap B_{i}\right|=\left|A_{i}\right|$.

It follows from (5.2) that $\left\{A_{1} \cap B_{1}, \ldots, A_{n} \cap B_{n}\right\}$ is a weak cardinal representation for $\langle E, \leq\rangle$. Moreover, if $1 \leq i \leq n$ and $A_{i}^{\prime} \subseteq A_{i} \cap B_{i}$ is such that $\left|A_{i}^{\prime}\right|=\left|A_{i} \cap B_{i}\right|=\left|A_{i}\right|$, then $\widehat{A}_{i}^{\prime} \supseteq B_{i}$ and so $A_{i}^{\prime}$ is cofinal in $A_{i} \cap B_{i}$.

Lemma 5.4. Let $\langle A, \leq\rangle$ be a quasi-ordered set with $|A|=\kappa \geq \omega$. If every subset of $A$ of cardinality $\kappa$ is cofinal in $A$, then $A$ contains a cofinal chain.

Proof. The hypothesis of the lemma implies that $|X|<\kappa$ for any antichain $X \subseteq A$ (since the removal of an element from $X$ results in a subset which is not cofinal in $A$ ). If $|A(\leq a)|=\kappa$ for some $a \in A$, then $\{a\}$ is a cofinal chain. Therefore, we may assume that $|A(\leq x)|<\kappa$ for every $x \in A$.

Note first that, for any cardinal $\mu<\kappa$, there is some element $b \in A$ such that $|A(\leq b)| \geq \mu$. Otherwise, by a set-mapping theorem of A. Hajnal [6], there is an antichain of size $\kappa$ in $A$, and this is a contradiction.

We claim that there is a subset $B \subseteq A$ of cardinality $\mathrm{cf} \kappa$ such that every subset of $B$ with cardinality $\mathrm{cf} \kappa$ is cofinal in $A$. If $\kappa$ is regular, the claim holds with $B=A$. Suppose that $\kappa$ is a singular cardinal. Choose cardinals $\kappa_{\alpha}<\kappa(\alpha<\operatorname{cf} \kappa)$ such that $\kappa=\sup \left\{\kappa_{\alpha}: \alpha<\operatorname{cf} \kappa\right\}$. By the above, for each $\alpha<\operatorname{cf} \kappa$, there is $b_{\alpha} \in A$ such that $\left|A\left(\leq_{\alpha}\right)\right| \geq \kappa_{\alpha}$. Put $B=\left\{b_{\alpha}: \alpha<\operatorname{cf} \kappa\right\}$. Then $|B|=\operatorname{cf} \kappa$, otherwise $\left|A\left(\leq b_{\alpha}\right)\right|=\kappa$ for some $\alpha$; also, if $B^{\prime} \subseteq B$ and $\left|B^{\prime}\right|=\operatorname{cf} \kappa$, then $B^{\prime}$ is cofinal in $A$ since it is cofinal in the set $\widehat{B}^{\prime}$ which has cardinality $\kappa$.

We now choose elements $x_{\alpha} \in B \quad(\alpha<\operatorname{cf} \kappa)$ as follows. Let $\alpha<\operatorname{cf} \kappa$ and suppose that $x_{\beta}$ has been chosen for $\beta<\alpha$. Put $C_{\beta}=\left\{b \in B: x_{\beta} \not b b\right\}$. 
Evidently, $C_{\beta}$ is not cofinal in $A$ and so $\left|C_{\beta}\right|<\operatorname{cf} \kappa$. Since $\mathrm{cf} \kappa$ is regular, it follows that there is $x_{\alpha} \in B \backslash \bigcup\left\{C_{\beta} \cup\left\{x_{\beta}\right\}: \beta<\alpha\right\}$. This defines a chain $C=\left\{x_{\alpha}: \alpha<\operatorname{cf} \kappa\right\}$ of size $\operatorname{cf} \kappa$ in $B$ which is therefore cofinal in $A$.

6. Closures satisfying ind $(\varphi) \leq k<\omega$. Proof of Theorem 2.4

In this section we prove Theorem 2.4 that: if a closure $\varphi$ on $E$ is such that $\operatorname{ind}(\varphi) \leq k<\omega$, then $\varphi$ has a cardinal representation, $\mathscr{A}$, of size $|\mathscr{A}| \leq k$, such that the cardinality of each member of $\mathscr{A}$ is either 1 or else a regular infinite cardinal.

Lemma 6.1. Let $\varphi$ be a preclosure on $E$ and suppose that ind $(\varphi) \leq \omega$. Then, for any set $C \subseteq E$, there are subsets $B, F \subseteq C$ such that $|B|=|C|, F$ is finite, and $\varphi\left(B^{\prime} \cup F\right) \supseteq B$ whenever $B^{\prime} \subseteq B$ and $\left|B^{\prime}\right|=|B|$.

Proof. Assume the contrary: there is a set $C \subseteq E$ such that, whenever $B, F \subseteq$ $C,|B|=|C|$, and $F$ is finite, there is a set $B^{\prime}=B^{\prime}(B, F) \subseteq B$ with $\left|B^{\prime}\right|=$ $|B|=|C|$ and $B \nsubseteq \varphi\left(B^{\prime} \cup F\right)$. Then we can define an infinite sequence

$$
B_{0}, B_{1}, x_{0}, B_{2}, x_{1}, \ldots, B_{n+1}, x_{n}, \ldots
$$

by putting $B_{0}=C, B_{n+1}=B^{\prime}\left(B_{n},\left\{x_{0}, \ldots, x_{n-1}\right\}\right)$, and choosing $x_{n} \in$ $B_{n} \backslash \varphi\left(B_{n+1} \cup\left\{x_{0}, x_{n-1}\right\}\right)$. Since $\left\{x_{i}: i \neq n\right\} \subseteq B_{n+1} \cup\left\{x_{0}, \ldots, x_{n-1}\right\}$, it follows that $\left\{x_{0}, x_{1}, x_{2}, \ldots\right\}$ is an infinite independent set, contradicting the assumption that $\operatorname{ind}(\varphi) \leq \omega$.

For a closure $\varphi$ on $E$ and a subset $A \subseteq E$, we define a closure $\varphi_{A}$ on $E \backslash \varphi(A)$ by setting $\varphi_{A}(X)=\varphi(A \cup X) \backslash \varphi(A)$ for $X \subseteq E \backslash \varphi(A)$; thus $\operatorname{dim}\left(\varphi_{A}\right)=$ $\min \{|X|: \varphi(X \cup A)=E\}$.

Lemma 6.2. Let $\varphi$ be a closure on $E$ and suppose that $\operatorname{ind}(\varphi) \leq \omega$. Let $A, D \subseteq E$ be such that $\varphi(A \cup D)=E$ and $|D|=\operatorname{dim}\left(\varphi_{A}\right)=\lambda \geq \omega$. Let $\kappa=\operatorname{cf} \lambda$. Then there are sets $F \subseteq D$ and $B \subseteq D \backslash \varphi(A \cup F)$ such that:

(i) $|B|=\kappa$ and $|F|<\omega$;

(ii) $|B \cap \varphi(A \cup X)|<\kappa$ whenever $X \subseteq D$ and $|X|<\kappa$;

(iii) $\varphi\left(B^{\prime} \cup F\right) \supseteq B$ whenever $B^{\prime} \subseteq B$ and $\left|B^{\prime}\right|=\kappa$.

Proof. Since $|D|=\lambda$ and $\operatorname{cf} \lambda=\kappa$, we can write $D=\bigcup\left\{D_{\alpha}: \alpha<\kappa\right\}$, where $\left|D_{\alpha}\right|<\lambda$ and $D_{\alpha} \subseteq D_{\beta}$ for $\alpha<\beta<\kappa$. If $X \subseteq D$ and $|X|<\kappa$, then $X \subseteq D_{\alpha}$ for some $\alpha<\kappa$.

Since $\left|D_{\alpha}\right|<\operatorname{dim}\left(\varphi_{A}\right)$ and $\varphi(A \cup D)=E$, and since $\varphi$ is a closure we have $\varphi\left(A \cup D_{\alpha}\right) \nsupseteq D$ for each $\alpha<\kappa$. Choose $c_{\alpha} \in D \backslash \varphi\left(A \cup D_{\alpha}\right)$ and let $C=\left\{c_{\alpha}: \alpha<\kappa\right\}$. Then $|C|=\kappa$, since $C \nsubseteq D_{\alpha}$ for all $\alpha<\kappa$.

By Lemma 6.1 there are sets $B, F \subseteq C \subseteq D$ satisfying (i) and (iii); we show that (ii) holds as well. Suppose $X \subseteq D$ and $|X|<\kappa$; then $X \subseteq D_{\alpha}$ for some $\alpha<\kappa$. It follows that $B \cap \varphi(A \cup X) \subseteq C \cap \varphi\left(A \cup D_{\alpha}\right) \subseteq\left\{c_{\beta}: \beta<\alpha\right\}$, and so $|B \cap \varphi(A \cup X)| \leq|\alpha|<\kappa$. 
Thus, we have sets $B, F \subseteq D$ satisfying (i), (ii), and (iii). It follows from (ii) that $|B \cap \varphi(A \cup F)|<\kappa$, whence $|B \backslash \varphi(A \cup F)|=\kappa$. Let $\widehat{B}=B \backslash \varphi(A \cup F)$; then $\widehat{B} \subseteq D \backslash \varphi(A \cup F)$, and (i)-(iii) still hold with $B$ replaced by $\widehat{B}$.

Lemma 6.3. Let $E \neq \varnothing$ and let $\varphi$ be a closure on $E$ such that ind $(\varphi)<\omega$. Then there are sets $A, B, F \subseteq E$ such that:

(*) $F \subseteq E \backslash \varphi(A), B \subseteq E \backslash \varphi(A \cup F),|F|<\omega$, and $|B|$ is either 1 or else a regular infinite cardinal;

$(* *) \varphi(A \cup B)=E$

$(* * *)$ whenever $A^{\prime} \subseteq A$ and $B^{\prime} \subseteq B$, we have $\varphi\left(A^{\prime} \cup B^{\prime} \cup F\right) \supseteq B \Leftrightarrow\left|B^{\prime}\right|=$ $|B|$.

Proof. For $A \subseteq E$ and $D \subseteq E \backslash \varphi(A)$, define

$$
i(A, D)=\max \left\{|X|: X \subseteq D \text { and } X \text { is } \varphi_{A} \text {-independent }\right\} ;
$$

of course, $i(A, D) \leq \operatorname{ind}\left(\varphi_{A}\right) \leq \operatorname{ind}(\varphi)<\omega$. Let

$$
\lambda=\min \left\{\operatorname{dim}\left(\varphi_{A}\right): A \subseteq E, \varphi(A) \neq E\right\},
$$

and let

$$
h=\min \left\{i(A, D): A \subseteq E, D \subseteq E \backslash \varphi(A), \varphi(A \cup D)=E,|D|=\operatorname{dim}\left(\varphi_{A}\right)=\lambda\right\} .
$$

Choose $A \subseteq E$ and $D \subseteq E \backslash \varphi(A)$ so that $\varphi(A \cup D)=E,|D|=\operatorname{dim}\left(\varphi_{A}\right)=\lambda$, and $i(A, D)=h$. Clearly, if $\lambda<\omega$, then $\lambda=1$ and we can simply take $B=D$ and $F=\varnothing$. Hence we may assume that $\lambda \geq \omega$; let $\kappa=\operatorname{cf} \lambda$. By Lemma 6.2, there are sets $F \subseteq D$ and $B \subseteq D \backslash \varphi(A \cup F)$ satisfying conditions (i)-(iii) of that lemma. Now $(*)$ and $(* * *)$ are easily verified; we will show that $(* *)$ also holds.

Suppose, on the contrary, that $\varphi(A \cup B) \neq E$. Let $\widehat{A}=A \cup B$ and $\widehat{D}=$ $D \backslash \varphi(A \cup B)$. Since $A \subseteq \widehat{A}$, we have $\operatorname{dim}\left(\varphi_{\widehat{A}}\right) \leq \operatorname{dim}\left(\varphi_{A}\right)=\lambda$; since $\varphi(\widehat{A}) \neq$ $E$, it follows by the minimality of $\lambda$ that $\operatorname{dim}\left(\varphi_{\widehat{A}}\right)=\lambda$. Since $\varphi(\widehat{A} \cup \widehat{D}) \supseteq$ $\varphi(\hat{A}) \cup \widehat{D}=\varphi(\widehat{A}) \cup D \supseteq A \cup D$, we have $\varphi(\widehat{A} \cup \widehat{D}) \supseteq \varphi(A \cup D)=E$. Hence $|\widehat{D}| \geq \operatorname{dim}\left(\varphi_{\widehat{A}}\right)=\lambda$; since $|\widehat{D}| \leq|D|=\lambda$, we have $|\widehat{D}|=\lambda$. Now, since $\widehat{A} \subseteq E, \widehat{D} \subseteq E \backslash \varphi(\widehat{A}), \varphi(\widehat{A} \cup \widehat{D})=E$, and $|\widehat{D}|=\operatorname{dim}\left(\varphi_{\widehat{A}}\right)=\lambda$, it follows by the minimality of $h$ that $i(\widehat{A}, \widehat{D}) \geq h$. Choose a $\varphi_{\widehat{A}}$-independent set $X \subseteq \widehat{D}$ with $|X|=h$. Since $X \subseteq \widehat{D} \subseteq D$ and $|X|=h<\omega \leq \kappa$, it follows by (ii) that $|B \cap \varphi(A \cup X)|<\kappa=|B|$. Choose $b \in B \backslash \varphi(A \cup X)$ and let $Y=X \cup\{b\}$. Since $A \cup\{b\} \subseteq \widehat{A}$ and $b \notin \varphi(A \cup X)$, it follows that $Y$ is a $\varphi_{A}$-independent subset of $D$; and $|Y|=h+1$ contradicting $i(A, D)=h$.

We will call $\mathscr{A}$ a regular representation of $\varphi$ if $\mathscr{A}$ is a cardinal representation of $\varphi$ and the cardinality of each member of $\mathscr{A}$ is either 1 or a regular infinite cardinal.

Lemma 6.4. Let $\varphi$ be a closure on $E$ such that $\operatorname{ind}(\varphi) \leq k<\omega$. Then there is a finite set $F \subseteq E^{\prime}$ such that $\varphi_{F}$ has a regular representation $\mathscr{A}$ with $|\mathscr{A}| \leq k$. 
Proof. For $k=0$ the result holds trivially. We now assume that $k>0$ and use induction on $k$. Choose sets $A, B, F \subseteq E$ as in Lemma 6.3. Put $A^{\prime}=$ $A \backslash \varphi(B \cup F)$ and define a closure $\varphi^{\prime}$ on $A^{\prime}$ by setting $\varphi^{\prime}(X)=\varphi(X \cup B \cup F) \cap A^{\prime}$ for $X \subseteq A^{\prime}$. If $X$ is a $\varphi^{\prime}$-independent subset of $A^{\prime}$ and if $b \in B$, then $X \cup\{b\}$ is $\varphi$-independent since $\varphi(A) \cap B=\varnothing$. Since $B \neq \varnothing$, it follows that $\operatorname{ind}\left(\varphi^{\prime}\right) \leq k-1$. By the induction hypothesis there is a finite set $F^{\prime} \subseteq A^{\prime}$ such that $\varphi_{F^{\prime}}^{\prime}$ has a regular representation $\mathscr{A}^{\prime}$ with $\left|\mathscr{A}^{\prime}\right| \leq k-1$. We will show that $\mathscr{A}=\mathscr{A}^{\prime} \cup\{B\}$ is a cardinal representation of $\varphi_{G}$, where $G=F \cup F^{\prime}$; since $|B|$ is either 1 or a regular infinite cardinal, it will follow that $\mathscr{A}$ is a regular representation.

Note that $\bigcup \mathscr{A} \subseteq E \backslash \varphi(G)$, since $\bigcup \mathscr{A}^{\prime} \subseteq A^{\prime} \backslash \varphi^{\prime}\left(F^{\prime}\right) \subseteq E \backslash \varphi(G)$ and $B \subseteq$ $E \backslash \varphi(A \cup F) \subseteq E \backslash \varphi(G)$. Consider any set $X \subseteq \cup \mathscr{A} ; X=B^{\prime} \cup Y$, where $B^{\prime}=X \cap B$ and $Y=X \backslash B=X \cap \bigcup \mathscr{A}^{\prime} \subseteq A^{\prime} \backslash \varphi^{\prime}\left(F^{\prime}\right)$. By the definition of $\varphi_{G}$, the equation

$$
\varphi_{G}(X)=E \backslash \varphi(G)
$$

means that $\varphi(G \cup X)=E$. Since $\varphi\left(A^{\prime} \cup B \cup F\right)=\varphi\left(A^{\prime} \cup \varphi(B \cup F)\right)=$ $\varphi(A \cup \varphi(B \cup F))=\varphi(A \cup B \cup F)=E,(6.1)$ is equivalent to

$$
\varphi(G \cup X) \supseteq B \quad \text { and } \quad \varphi(G \cup X) \supseteq A^{\prime} .
$$

If $\varphi(G \cup X) \supseteq B$, then

$$
\varphi(G \cup X)=\varphi(G \cup X \cup B)=\varphi(G \cup Y \cup B)=\varphi\left(F^{\prime} \cup Y \cup B \cup F\right) ;
$$

hence (6.2) is equivalent to

$$
\varphi(G \cup X) \supseteq B \quad \text { and } \quad \varphi\left(F^{\prime} \cup Y \cup B \cup F\right) \supseteq A^{\prime} .
$$

Since $\varphi^{\prime}\left(F^{\prime} \cup Y\right)=\varphi\left(F^{\prime} \cup Y \cup B \cup F\right) \cap A^{\prime},(6.3)$ is equivalent to

$$
\varphi(G \cup X) \supseteq B \quad \text { and } \quad \varphi^{\prime}\left(F^{\prime} \cup Y\right)=A^{\prime} \text {, }
$$

which in turn is equivalent to

$$
\varphi(G \cup X) \supseteq B \quad \text { and } \quad \varphi_{F^{\prime}}^{\prime}(Y)=A^{\prime} \backslash \varphi\left(F^{\prime}\right) .
$$

Since $G \cup X=\left(F^{\prime} \cup Y\right) \cup B^{\prime} \cup F$, where $F^{\prime} \cup Y \subseteq A$ and $B^{\prime} \subseteq B$, it follows by $(* * *)$ that $\varphi(G \cup X) \supseteq B$ if and only if $\left|B^{\prime}\right|=|B|$. From this and the fact that $\mathscr{A}^{\prime}$ is a cardinal representation of $\varphi_{F^{\prime}}^{\prime}$ and $Y \subseteq \cup \mathscr{A}^{\prime}$, it follows that (6.5) is equivalent to

$$
\left|B^{\prime}\right|=|B| \text { and }|Y \cap T|=|T| \text { for each } T \in \mathscr{A}^{\prime} .
$$

Since $B^{\prime}=X \cap B$ and $Y \cap T=X \cap T$ for $T \in \mathscr{A}^{\prime}$, the equivalence of (6.1) to (6.6) shows that $\mathscr{A}$ is a cardinal representation of $\varphi_{G}$.

Proof of Theorem 2.4. Let $\varphi$ be a closure on $E$ and suppose that $\operatorname{ind}(\varphi) \leq$ $k<\omega$. By Lemma 6.4 there is a minimal finite set $F \subseteq E$ such that $\varphi_{F}$ has a regular representation $\mathscr{A}$ with $|\mathscr{A}| \leq k$. By Theorem 2.10 , we may assume that the members of $\mathscr{A}$ are pairwise disjoint. We want to show that $F=\varnothing$. 
Suppose $F \neq \varnothing$, say $x \in F$. Put $F^{\prime}=F \backslash\{x\}$. Because of the minimality of $F, \mathscr{A}$ is not a cardinal representation of $\varphi_{F^{\prime}}$, and so there is a set $X \subseteq$ $\cup \mathscr{A}$ such that $|X \cap A|=|A|$ for each $A \in \mathscr{A}$, and $x \notin \varphi\left(F^{\prime} \cup X\right)$. It follows that $\{X \cap A: A \in \mathscr{A}\} \cup\{x\}$ is a regular representation of $\varphi_{F^{\prime}}$, and so $|\mathscr{A}|=k$. Since $\mathscr{A}$ is a cardinal representation of $\varphi_{F}$, and since $X \subseteq \cup \mathscr{A}$ and $|X \cap A|=|A|>0$ for each $A \in \mathscr{A}$, it follows that $X \cap A \nsubseteq \varphi_{F}(X \backslash A)$; choose $x_{A} \in(X \cap A) \backslash \varphi_{F}(X \backslash A)=(X \cap A) \backslash \varphi(F \cup(X \backslash A))$ for each $A \in \mathscr{A}$. Then, since the members of $\mathscr{A}$ are pairwise disjoint, $\{x\} \cup\left\{x_{A}: A \in \mathscr{A}\right\}$ is a $\varphi$-independent set of cardinality $k+1$. This contradiction proves that $F=\varnothing$, and the theorem follows.

We conclude this section by deducing three corollaries from Theorem 2.4. The first is a trivial self-strengthening of Theorem 2.4; the second is a result of E. C. Milner and M. Pouzet [9].

Corollary 6.5. Let $\varphi$ be a closure on $E$ such that $\operatorname{ind}(\varphi) \leq k<\omega$. If $E^{\prime} \subseteq E$ and $\varphi\left(E^{\prime}\right)=E$, then $\varphi$ has a regular representation $\mathscr{A}$ such that $|\mathscr{A}| \leq k$ and $\bigcup \mathscr{A} \subseteq E^{\prime}$.

Proof. Apply Theorem 2.4 to the closure $\varphi^{\prime}$ on $E^{\prime}$ defined by setting $\varphi^{\prime}(X)=$ $\varphi(X) \cap E^{\prime}$ for $X \subseteq E^{\prime}$.

Corollary 6.6 [9]. If $\varphi$ is a closure on $E$ and $\operatorname{ind}(\varphi)<\omega$, then $\operatorname{dim}(\varphi)$ is either finite, or else it is a regular infinite cardinal.

Proof. Choose $E^{\prime} \subseteq E$ so that $\varphi\left(E^{\prime}\right)=E$ and $\left|E^{\prime}\right|=\operatorname{dim}(\varphi)$. By Corollary $6.5, \varphi$ has a finite regular representation $\mathscr{A}$ such that $\bigcup \mathscr{A} \subseteq E^{\prime}$; let $X=$ $\cup \mathscr{A}$. Since $\varphi(X)=E$ and $X \subseteq E^{\prime}$, it follows that $|X|=\operatorname{dim}(\varphi)$. Clearly $|X|$ is either finite or else a regular infinite cardinal.

Corollary 6.7. Let $\varphi$ be a closure on $E$ such that $\operatorname{ind}(\varphi)<\omega$, and let $\mathscr{A}$ be any (finite or infinite) cardinal representation of $\varphi$. Then the cardinality of any infinite member of $\mathscr{A}$ is a regular cardinal.

Proof. By Corollary 6.5 , the closure $\varphi$ has a finite regular representation $\mathscr{B}=$ $\left\{B_{1}, \ldots, B_{k}\right\}$ such that $\bigcup \mathscr{B} \subseteq \cup \mathscr{A}$. Consider any $A \in \mathscr{A}$. For each $i \in$ $\{1, \ldots, k\}$, choose $B_{i}^{\prime} \subseteq B_{i}$ so that $\left|B_{i}^{\prime}\right|=\left|B_{i}\right|$ and either $\left|B_{i}^{\prime} \cap A\right| \leq 1$ or else $B_{i}^{\prime} \subseteq A$. Let $X=\bigcup\left\{B_{i}^{\prime}: 1 \leq i \leq k\right\}$. Since $X \subseteq \bigcup \mathscr{B} \subseteq \bigcup \mathscr{A}$ and $\varphi(X)=E$, it follows that $|X \cap A|=|A|$. Since $|A|=|X \cap A|=\left|\bigcup\left\{B_{i}^{\prime} \cap A: 1 \leq i \leq k\right\}\right|$, and since $\left|B_{i}^{\prime} \cap A\right|$ is either finite or a regular infinite cardinal for each $i$, the same is true of $|A|$.

\section{AN APPLICATION TO POSETS OF FINITE BREADTH}

Theorem 7.1 below, concerning the gaps in a partially ordered set, is a fairly simple application of Theorem 2.4. This provides a new proof of a theorem of D. Duffus and M. Pouzet [4, Corollary 7.2].

Let $\mathscr{P}=\langle P, \leq\rangle$ be a partially ordered set. For $X, Y \subseteq P$, we write $X \leq Y$ if and only if $x \leq y$ holds whenever $x \in X$ and $y \in Y$. Also, for $X \subseteq P$, 
put $X^{+}=\{y \in P: X \leq\{y\}\}$ and $X^{-}=\{y \in P:\{y\} \leq X\}$. Thus, in particular, we have $\varnothing^{+}=\varnothing^{-}=P$. A gap in $\mathscr{P}$ is a pair $(A, B)$ of subsets of $P$ such that $A \leq B$ and $A^{+} \cap B^{-}=\varnothing$ (i.e., there is no element $x \in P$ such that $A \leq\{x\} \leq B)$. A subgap of $(A, B)$ is a gap $\left(A^{\prime}, B^{\prime}\right)$ with $A^{\prime} \subseteq A$ and $B^{\prime} \subseteq B$. The gap $(A, B)$ is irreducible if $\left|A^{\prime}\right|=|A|$ and $\left|B^{\prime}\right|=|B|$ for every subgap $\left(A^{\prime}, B^{\prime}\right)$. A gap $(A, B)$ is called regular if $|A|$ and $|B|$ are both regular infinite cardinals or if one of them is a regular infinite cardinal and the other is 0 .

We define the breadth of a partially ordered set $\mathscr{P}=\langle P, \leq\rangle$ to be $b(\mathscr{P})=$ $\sup \left\{|X|: X \subseteq P\right.$ and $\left.(\forall Y \subseteq X)\left(Y \neq X \Rightarrow Y^{+} \neq X^{+}\right)\right\}$. (This definition agrees, for example, with that given in Birkhoff [1] for finite lattices.) The one-sidedness of the definition is only apparent; we can replace $Y^{+} \neq X^{+}$by $Y^{-} \neq X^{-}$, so that the dual partial order $\mathscr{P}^{d}=\langle P, \geq\rangle$ has the same breadth as $\mathscr{P}$. (Suppose $\mu<b(\mathscr{P})$. Then there is $X \subseteq P$ such that $|X|>\mu$ and $(X \backslash\{x\})^{+} \neq X^{+}$for each $x \in X$. Choose $x^{\prime} \in(X \backslash\{x\})^{+} \backslash X^{+} \quad(x \in X)$ and put $X^{\prime}=\left\{x^{\prime}: x \in X\right\}$. Then $\left|X^{\prime}\right|=|X|>\mu$ and $Y^{-} \neq\left(X^{\prime}\right)^{-}$for every proper subset $Y \subseteq X^{\prime}$, so that $\mu<b\left(\mathscr{P}^{d}\right)$, etc.)

Theorem 7.1. Let $(A, B)$ be a gap in the partially ordered set $\mathscr{P}$ of finite breadth $k$. Then there are nonnegative integers $m, n$ and pairwise disjoint subsets $A_{1}, \ldots, A_{m} \subseteq A$ and $B_{1}, \ldots, B_{n} \subseteq B$ such that:

(i) each $\left|A_{i}\right|,\left|B_{j}\right| \quad(1 \leq i \leq m, 1 \leq j \leq n)$ is either 1 or a regular infinite cardinal;

(ii) for $A^{\prime} \subseteq \bigcup_{1 \leq i \leq m} A_{i}$ and $B^{\prime} \subseteq \bigcup_{1 \leq j \leq n} B_{j}$,

$$
\left(A^{\prime}, B^{\prime}\right) \text { is a gap } \Leftrightarrow \begin{cases}\left|A^{\prime} \cap A_{i}\right|=\left|A_{i}\right| & (1 \leq i \leq m) \text { and } \\ \left|B^{\prime} \cap B_{j}\right|=\left|B_{j}\right| & (1 \leq j \leq n)\end{cases}
$$

(iii) $m \leq k$ and $n \leq k$;

(iv) $m, n \leq 1$ if $\mathscr{P}$ is a lattice.

Corollary 7.2 [4]. A gap in a lattice of finite breadth contains a regular, irreducible subgap.

Proof of Theorem 7.1. For $X \subseteq A \cup B$ define

$$
\begin{gathered}
\psi(X)=(X \cap A)^{+} \cap(X \cap B)^{-}, \\
\varphi(X)=\left((\psi(X))^{-} \cap A\right) \cup\left((\psi(X))^{+} \cap B\right) .
\end{gathered}
$$

Then $\varphi$ is a closure on $A \cup B$. (Remark: Our definition of a closure requires that $\varphi(\varnothing)=\varnothing$, but here $\varphi(\varnothing)=\left(P^{-} \cap A\right) \cup\left(P^{+} \cap B\right)$ may be nonempty, e.g. if $P$ has a least element which belongs to $A$. However, without loss of generality we can assume that the extremal elements of $P$, if they exist, do not belong to $A \cup B$ since $\left(A \backslash P^{-}, B \backslash P^{+}\right)$is a subgap of $(A, B)$.)

Note that, for $X \subseteq A \cup B,(X \cap A, X \cap B)$ is a gap if and only if $\psi(X)=\varnothing$. If $\psi(X)=\varnothing$, then $\varphi(X)=A \cup B$. Also, if $x \in \psi(X) \neq \varnothing$, then either $A \not\{\{x\}$ 
or $\{x\} \not \subset B$ and, in either case, $\varphi(X) \neq A \cup B$. Thus, for $X \subseteq A \cup B$, we have

$$
(X \cap A, X \cap B) \text { is a gap } \Leftrightarrow \varphi(X)=A \cup B .
$$

Since the breadth of $\mathscr{P}$ is $k$, it follows that, whenever $Z \subseteq A$ and $|Z|>k$, there is some $z \in Z$ such that $(Z \backslash\{z\})^{+}=Z^{+}$and hence $z \in \varphi(Z \backslash\{z\})$. It follows that there is no $\varphi$-independent subset of $A$ of size $k+1$; similarly for $B$. Therefore $\operatorname{ind}(\varphi) \leq 2 k$ and so, by Theorem 2.4 , there are subsets $A_{1}, \ldots, A_{m} \subseteq A, B_{1}, \ldots, B_{n} \subseteq B$ such that $m+n \leq 2 k$ and $\mathscr{A}=\left\{A_{1}, \ldots, A_{m}, B_{1}, \ldots, B_{n}\right\}$ is a cardinal representation for $\varphi$ such that (i) holds. By Theorem 2.10 we may assume that the members of $\mathscr{A}$ are pairwise disjoint. Also, (ii) is an immediate consequence of the equivalence (7.1) and the fact that $\left\{A_{1}, \ldots, A_{m}, B_{1}, \ldots, B_{n}\right\}$ is a cardinal representation of $\varphi$. By (ii), for each $i \quad(1 \leq i \leq m),\left(\bigcup_{h \neq i} A_{h}, \bigcup_{j} B_{j}\right)$ is not a gap, and so there is $y_{i} \in P$ such that $\bigcup_{h \neq i} A_{h} \leq\left\{y_{i}\right\} \leq \bigcup_{j} B_{j}$. Therefore, $A_{i} \mathbb{Z}\left\{y_{i}\right\}$ and so there is $x_{i} \in A_{i}$ such that $x_{i} \not \leq y_{i}$. Let $X=\left\{x_{i}: 1 \leq i \leq m\right\}$; then $|X|=m$ and $\left(X^{\prime}\right)^{+} \neq X^{+}$for each proper subset $X^{\prime}$ of $X$, and therefore $m \leq k$. Similarly, $n \leq k$, and so (iii) holds.

It remains to prove (iv). Assume $\mathscr{P}$ is a lattice. Suppose $m>1$. Then we may assume that $A_{1}, A_{2}$ are both nonempty. Since $\left(\bigcup_{i \neq 1} A_{i}, \bigcup_{j} B_{j}\right)$ is not a gap by (ii), it follows that there is $x_{1} \in P$ such that $\bigcup_{i \neq 1} A_{i} \leq\left\{x_{1}\right\} \leq \bigcup_{j} B_{j}$. Similarly, there is $x_{2} \in P$ such that $\bigcup_{i \neq 2} A_{i} \leq\left\{x_{2}\right\} \leq \bigcup_{j} B_{j}$. If $x$ is the join of $x_{1}$ and $x_{2}$ in the lattice, it follows that $\bigcup_{i} A_{i} \leq\{x\} \leq \bigcup_{j} B_{j}$ and this is a contradiction since $\left(\bigcup_{i} A_{i}, \bigcup_{j} B_{j}\right)$ is a gap by (ii). Therefore, $m \leq 1$, and similarly $n \leq 1$.

\section{Preclosures on $\omega$ and a Proof of Theorem 2.5}

We begin this section by establishing some necessary conditions for a preclosure to have a cardinal representation, which are also sufficient in the case of preclosures on a denumerable set. For a preclosure $\varphi$ on a set $E$, we define the kernel of a set $G \subseteq E$ to be the set $\mathfrak{k}_{\varphi}(G)=\{x \in G: \varphi(G \backslash\{x\}) \neq \varphi(G)\}$ $(=\bigcap\{X: X \subseteq G$ and $\varphi(X)=\varphi(G)\})$.

Lemma 8.1. For a preclosure $\varphi$ on $E$ and subset $G \subseteq E$, the following statements are equivalent:

$$
\begin{aligned}
& \text { Whenever } \mathfrak{k}_{\varphi}(G) \subseteq H \subseteq G \text { and } \varphi(H) \neq \varphi(G) \text {, then } \varphi(H \cup F) \neq \\
& \varphi(G) \text { for every finite set } F \subseteq G .
\end{aligned}
$$

$$
\mathfrak{k}_{\varphi}(H)=\mathfrak{k}_{\varphi}(G) \text { for every set } H \subseteq G \text { such that } \varphi(H)=\varphi(G) \text {. }
$$

Proof. $(8.1) \Rightarrow(8.2)$ Suppose (8.1) holds. Let $H \subseteq G, \varphi(H)=\varphi(G)$, and suppose that $\mathfrak{k}_{\varphi}(H) \neq \mathfrak{k}_{\varphi}(G)$. Since $H \supseteq \mathfrak{k}_{\varphi}(H) \supseteq \mathfrak{k}_{\varphi}(G)$, there is some element $x \in \mathfrak{k}_{\varphi}(H) \backslash \mathfrak{k}_{\varphi}(G)$. Since $\varphi(H \backslash\{x\}) \neq \varphi(G)=\varphi(H)$, it follows that $\mathfrak{k}_{\varphi}(G) \nsubseteq$ $H \backslash\{x\}$ and this is a contradiction. Therefore (8.2) holds.

$(8.2) \Rightarrow(8.1)$ Suppose that (8.2) holds. Let $H \subseteq G, \varphi(H) \neq \varphi(G)$, and suppose that $\varphi(H \cup F)=\varphi(G)$ for some finite $F \subseteq G$. We can assume that $F$ 
is minimal with this property. But then $F \subseteq \mathfrak{k}_{\varphi}(H \cup F)=\mathfrak{k}_{\varphi}(G)$ and $F \nsubseteq H$, and so $\mathfrak{k}_{\varphi}(G) \nsubseteq H$. Thus (8.1) holds.

Theorem 8.2. If the preclosure $\varphi$ has a cardinal representation then there is a generating set $G$ which satisfies the condition (8.2) (or 8.1).

Proof. Suppose that $\mathscr{A}$ is a cardinal representation of $\varphi$. We claim the generating set $G=\bigcup \mathscr{A}$ satisfies the condition (8.2). For this we need only show that, whenever $H \subseteq G$ is a generating set, then $\mathfrak{k}_{\varphi}(H) \subseteq \mathfrak{k}_{\varphi}(G)$. Let $x \in \mathfrak{k}_{\varphi}(H)$. Since $\varphi(H \backslash\{x\}) \neq \varphi(H)$, there is some $A \in \mathscr{A}$ such that $|(H \backslash\{x\}) \cap A|<|A|=|H \cap A|$. Thus $A$ is a finite set containing the element $x$. But then $|(G \backslash\{x\}) \cap A|<|A|$ and, since $\mathscr{A}$ is a cardinal representation, it follows that $x \in \mathfrak{k}_{\varphi}(G)$.

Theorem 8.3. The preclosure $\varphi$ on $\omega$ has a cardinal representation if and only if there is a generating set $G$ such that (8.2) holds.

Proof. The necessity follows from Theorem 8.2 , we have only to prove the sufficiency. Suppose that $G$ is a generating set which satisfies (8.2). We will show that

$$
\mathscr{A}=\{A \subseteq G:(\forall X \subseteq G)(\varphi(X)=\omega \Rightarrow|X \cap A|=|A|)\}
$$

is a cardinal representation for $\varphi$. To see this, consider any set $Y \subseteq \cup \mathscr{A} \subseteq G$. If $\varphi(Y)=\omega$, then obviously $|Y \cap A|=|A|$ for each $A \in \mathscr{A}$. Now suppose conversely that $|Y \cap A|=|A|$ for each $A \in \mathscr{A}$. Then $\mathfrak{k}_{\varphi}(G) \subseteq Y$ since $\{x\} \in \mathscr{A}$ for each $x \in \mathfrak{k}_{\varphi}(G)$. Suppose for a contradiction that $\varphi(Y) \neq \omega$. Then $G \backslash Y \neq \varnothing$ and so $G \backslash Y \notin \mathscr{A}$ (since $|Y \cap(G \backslash Y)|<|G \backslash Y|$ ). Hence there is $X \subseteq G$ such that $\varphi(X)=\omega$ and $|X \cap(G \backslash Y)|<|G \backslash Y|$. Therefore, $F=X \cap(G \backslash Y)$ is finite. Now we have that $\varphi(Y \cup F) \supseteq \varphi(X)=\varphi(G)$, and this contradicts $(8.1)$ since $\mathfrak{k}_{\varphi}(G) \subseteq Y \subseteq G$ and $\varphi(Y) \neq \varphi(G)$.

We use Theorem 8.3 to prove Theorem 2.5.

Proof of Theorem 2.5. Suppose that $\varphi$ is a closure on $\omega$ and that $\varphi$ has no cardinal representation. Then by Theorem 8.3 , for any generating set $G$, there is a generating set $H$ such that $\mathfrak{k}_{\varphi}(G) \subset \mathfrak{k}_{\varphi}(H) \subset H \subset G$, where $\subset$ denotes strict inclusion. It follows that there is a strictly decreasing sequence of generating sets $G_{n}(n \in \omega)$ such that $K_{n}=\mathfrak{k}_{\varphi}\left(G_{n}\right)$ is strictly increasing. Then $K=\bigcup\left\{K_{n}\right.$ : $n \in \omega\}$ is an infinite independent set since, for any $x \in K, \varphi\left(G_{n} \backslash\{x\}\right) \neq \omega$ for some $n$ and so $x \notin \varphi\left(G_{n} \backslash\{x\}\right) \supseteq \varphi(K \backslash\{x\})$ because $\varphi$ is a closure.

\section{Algebraic closures on $\omega$ with $\operatorname{ind}(\varphi)<\omega$}

In this section we give a proof of Theorem 2.6 and we also prove an easy self-strengthening, Theorem 9.6. We need several preliminary lemmas. The first lemma, Lemma 9.1, has an independent interest; the proof employs an argument similar to one used by P. Erdös and A. Tarski in [5]. 
Lemma 9.1. Let $\varphi$ be a closure on $E$ and suppose that ind $(\varphi) \leq \omega$. Then there are finite sets $F \subseteq E$ and $\mathscr{A} \subseteq \mathscr{P}(E)$ such that (i) $\varphi(F \cup \cup \mathscr{A})=E$ and (ii) for each $A \in \mathscr{A}$, and every finite set $G \supseteq F, N(G, A)=\{X \subseteq A: \varphi(G \cup X) \unrhd A\}$ is an ideal in $\mathscr{P}(A)$.

Proof. For finite sets $F \subseteq E$ and $\mathscr{A} \subseteq \mathscr{P}(E)$ let us say that a set $B \subseteq E$ is $(F, \mathscr{A})$-good if $\varphi(F \cup \cup \mathscr{A}) \supseteq B$, and $N(G, A)$ is an ideal in $\mathscr{P}(A)$ whenever $A \in \mathscr{A}$ and $G$ is a finite set such that $F \subseteq G \subseteq \omega$. We say that $B$ is good if it is $(F, \mathscr{A})$-good for some $F$ and $\mathscr{A}$; otherwise $B$ is bad.

Claim. For every bad set $B \subseteq E$ and every finite set $F \subseteq \omega$, there is a bad set $B^{\prime} \subseteq B$ such that $B \nsubseteq \varphi\left(F \cup B^{\prime}\right)$.

Proof of claim. Since $B$ is not $(F,\{B\})$-good, there is a finite set $G$ such that $F \subseteq G \subseteq E$ and $N(G, B)$ is not an ideal in $\mathscr{P}(B)$. But $\varnothing \in N(G, B)$ since $B$ is not $(G, \varnothing)$-good; hence there are $B_{1}, B_{2} \in N(G, B)$ such that $B_{1} \cup B_{2} \notin N(G, B)$. If, for $i \in\{1,2\}, B_{i}$ is $\left(F_{i}, \mathscr{A}_{i}\right)$-good, then $B$ is $\left(G \cup F_{1} \cup\right.$ $\left.F_{2}, \mathscr{A}_{1} \cup \mathscr{A}_{2}\right)$-good (since $\left.\varphi\left(G \cup F_{1} \cup F_{2} \cup \cup\left(\mathscr{A}_{1} \cup \mathscr{A}_{2}\right)\right) \supseteq \varphi\left(G \cup B_{1} \cup B_{2}\right) \supseteq B\right)$, which is a contradiction. Therefore, $B_{i}$ is bad for some $i \in\{1,2\}$, and $B \nsubseteq \varphi\left(F \cup B_{i}\right)$ since $B_{i} \in N(G, B)$ and $F \subseteq G$.

To prove the lemma, we need to show that $E$ is good. Suppose $E$ is bad. Then by the above claim we can construct $E_{n}, x_{n} \quad(n<\omega)$ so that $E=E_{0} \supseteq$ $E_{1} \supseteq \cdots \supseteq E_{n} \supseteq \cdots, E_{n}$ is bad, and $x_{n} \in E_{n} \backslash \varphi\left(\left\{x_{0}, \ldots, x_{n-1}\right\} \cup E_{n+1}\right)$. Then $\left\{x_{n}: n<\omega\right\}$ is an infinite independent set, which contradicts the hypothesis that $\operatorname{ind}(\varphi) \leq \omega$.

We need some new definitions. As usual for a set $X$ and a cardinal $\kappa$ we write $[X]^{\kappa}=\{Y \subseteq X:|Y|=\kappa\},[X]^{<\kappa}=\{Y \subseteq X:|Y|<\kappa\}$. Let $\mathscr{S} \subseteq[\omega]^{<\omega}$. We denote by $\overline{\mathscr{S}}$ the set $\{X \subseteq \omega: X \supseteq S$ for some $S \in \mathscr{S}\}$. Also, for $F \subseteq \omega$, we define $\mathscr{S}-F=\{S \backslash F: S \in \mathscr{S}\}$ (where, as usual, $A \backslash B$ denotes $\{x \in A: x \notin B\})$. We will say that $\mathscr{S}$ blocks the set $M \subseteq \omega$ if and only if $[M]^{\omega} \subseteq \overline{\mathscr{S}}$. In particular, $\mathscr{S}$ blocks any finite subset of $\omega$; also, if $\mathscr{S}$ blocks $M$, then $\mathscr{S}$ blocks any set $A \subseteq \omega$ such that $A \backslash M$ is finite. For an ordinal $\alpha$, we say that $\mathscr{S} \alpha$-blocks $M$ if, and only if,

EITHER (i) $\alpha=0$ and $\varnothing \in \mathscr{S}$,

OR (ii) $\alpha>0$ and for all but finitely many elements $x \in M$, there is $\beta=\beta(x)<\alpha$ such that $\mathscr{S}-\{x\} \beta$-blocks $M$.

The following statements are easy consequences of the above inductive definition of " $\alpha$-blocks $M$."

$$
\begin{aligned}
& \text { If } M \subseteq \omega, \mathscr{S} \subseteq[\omega]^{<\omega}, \alpha<\alpha^{\prime}, \text { then } \\
& \mathscr{S} \alpha \text {-blocks } M \Rightarrow \mathscr{S} \alpha^{\prime} \text {-blocks } M .
\end{aligned}
$$

If $M, M^{\prime} \subseteq \omega,\left|M^{\prime} \backslash M\right|<\omega, \mathscr{S} \subseteq[\omega]^{<\omega}$, then

$\mathscr{S} \alpha$-blocks $M \Rightarrow \mathscr{S} \alpha$-blocks $M^{\prime}$. 


$$
\begin{aligned}
& \text { If } M^{\prime} \subseteq M \subseteq \omega \text { and } \mathscr{S}, \mathscr{S}^{\prime} \subseteq[\omega]^{<\omega}, \mathscr{S} \cap\left[M^{\prime}\right]^{<\omega} \subseteq \mathscr{S}^{\prime} \text {, then } \\
& \mathscr{S} \alpha \text {-blocks } M \Rightarrow \mathscr{S}^{\prime} \alpha \text {-blocks } M^{\prime} \text {. }
\end{aligned}
$$

Lemma 9.2. For $M \subseteq \omega$ and $\mathscr{S} \subseteq[\omega]^{<\omega}, \mathscr{S}$ blocks $M \Leftrightarrow\left(\exists \alpha<\omega_{1}\right) \mathscr{S} \alpha$ blocks $M$.

Proof. $(\Rightarrow)$ Suppose $\mathscr{S}$ blocks $M$ and suppose for contradiction that $\mathscr{S}$ does not $\alpha$-block $M$ for any $\alpha<\omega_{1}$. Then, $\varnothing \notin \mathscr{S}$ and, for $0<\alpha<\omega_{1}$, there is $x(\alpha) \in M$ such that $\mathscr{S}-\{x(\alpha)\}$ does not $\beta$-block $M$ for any $\beta<\alpha$. There is $x_{0} \in M$ such that $x(\alpha)=x_{0}$ for $\omega_{1}$ values of $\alpha$ and so $\mathscr{S}-\left\{x_{0}\right\}$ does not $\beta$-block $M$ for any $\beta<\omega_{1}$. Repeating this argument it follows that there are distinct elements $x_{n} \in M(n<\omega)$ such that $\mathscr{S}-\left\{x_{0}, \ldots, x_{n-1}\right\}$ does not $\alpha$-block $M$ for any $n<\omega$ and $\alpha<\omega_{1}$. Since $\mathscr{S}$ blocks $M$, there are $n<\omega$ and $S \in \mathscr{S}$ such that $S \subseteq\left\{x_{0}, \ldots, x_{n-1}\right\}$. But this implies that $\varnothing \in$ $\mathscr{S}-\left\{x_{0}, \ldots, x_{n-1}\right\}$ and we have the contradiction that $\mathscr{S}-\left\{x_{0}, \ldots, x_{n-1}\right\}$ 0-blocks $M$.

$(\Leftarrow)$ We show by induction on $\alpha$ that, if $\mathscr{S}$ does not block $M$, then $\mathscr{S}$ does not $\alpha$-block $M$. For $\alpha=0$ this is clear. Suppose $\alpha>0$. Since $\mathscr{S}$ does not block $M$, there is an infinite set $M^{\prime} \subseteq M$ such that $M^{\prime} \notin \overline{\mathscr{S}}$. Therefore, $\mathscr{S}-\{x\}$ does not block $M$ for $x \in M^{\prime}$ and so, by the induction hypothesis, $\mathscr{S}$ does not $\alpha$-block $M$.

Note that, if $\varphi$ is an algebraic preclosure on $\omega$, then

$$
\operatorname{ind}(\varphi) \leq \omega \Leftrightarrow \operatorname{DEP}(\varphi) \text { blocks } \omega,
$$

where $\operatorname{DEP}(\varphi)=\{X \subseteq \omega: X$ is finite and $\varphi$-dependent $\}$.

We shall write $\varphi \in$ FCR to abbreviate the assertion that the preclosure $\varphi$ has a finite cardinal representation. Also, we write $\varphi \in \mathrm{AC}$, if $\varphi$ is an algebraic closure on $\omega$. Let $\mathbf{P}(\alpha), \mathbf{Q}(\alpha)$, and $\mathbf{R}$ denote the following statements:

$\mathbf{P}(\alpha): \varphi \in \mathrm{AC}$ and $\operatorname{DEP}(\varphi) \alpha$-blocks $\omega \Rightarrow \varphi \in \mathrm{FCR}$.

$\mathbf{Q}(\alpha): \varphi \in \mathrm{AC}, \operatorname{DEP}(\varphi) \alpha$-blocks $\omega$ and $\{X \subseteq \omega: \varphi(X) \neq \omega\}$ is an ideal $\Rightarrow \varphi \in$ FCR.

$\mathbf{R}: \varphi \in \mathrm{AC}$ and $\operatorname{ind}(\varphi) \leq \omega \Rightarrow \varphi \in \mathrm{FCR}$.

We want to prove Theorem 2.6 which asserts that $\mathbf{R}$ is true. To this end notice that, by Lemma 9.2 and (9.4), we have the equivalence:

$$
\mathbf{R} \Leftrightarrow\left(\forall \alpha<\omega_{1}\right) \mathbf{P}(\alpha) .
$$

We will use (9.5) and the following lemma to prove $\mathbf{R}$.

Lemma 9.3. For any ordinal $\alpha<\omega_{1}, \mathbf{P}(\alpha) \Leftrightarrow \mathbf{Q}(\alpha)$.

Proof. Clearly $\mathbf{P}(\alpha) \Rightarrow \mathbf{Q}(\alpha)$, we have to prove the converse. We assume $\mathbf{Q}(\alpha)$ is true. Let $\varphi \in \mathrm{AC}$ and suppose that $\operatorname{DEP}(\varphi) \alpha$-blocks $\omega$. By Lemma 9.2 and the equivalence (9.4), it follows that $\operatorname{ind}(\varphi) \leq \omega$ and so, by Lemma 9.1, there are $F \in[\omega]^{<\omega}$ and $\mathscr{A} \in[\mathscr{P}(\omega)]^{<\omega}$ such that $\varphi(F \cup \bigcup \mathscr{A})=\omega$ and $N(F, A)=\{X \subseteq A: A \nsubseteq \varphi(F \cup X)\}$ is an ideal for each $A \in \mathscr{A}$. For $A \in \mathscr{A}$, let $\varphi_{A}$ be the closure on $A$ defined by $\varphi_{A}(Y)=\varphi(Y \cup F) \cap A \quad(\varnothing \neq Y \subseteq A)$. If 
$A \in \mathscr{A}$ is finite, then obviously $\varphi_{A} \in \mathrm{FCR}$. Suppose $A \in \mathscr{A}$ is infinite. Then, since $\operatorname{DEP}(\varphi) \cap[A]^{\omega} \subseteq \operatorname{DEP}\left(\varphi_{A}\right)$, it follows by (9.3) that $\operatorname{DEP}\left(\varphi_{A}\right) \alpha$-blocks $A$ and so $\varphi_{A} \in \mathrm{FCR}$ by $\mathbf{Q}(\alpha)$. Thus, for each $A \in \mathscr{A}$, the closure $\varphi_{A}$ has a finite cardinal representation. Since $F$ and $\mathscr{A}$ are both finite, and since $\varphi(F \cup \bigcup \mathscr{A})=\omega$, it follows that there is a finite weak cardinal representation of $\varphi$. Hence $\varphi \in$ FCR by Theorem 2.8 .

We will say that an ideal $\mathscr{I} \subseteq \mathscr{P}(\omega)$ is nice if there are sets $\mathscr{G}_{n} \subseteq[\omega]^{<\omega}$ $(n<\omega)$ such that

$$
\mathscr{P}(\omega) \backslash \mathscr{I}=\bigcap_{n<\omega} \overline{\mathscr{G}}_{n} .
$$

Lemma 9.4. If $\varphi$ is an algebraic preclosure on $\omega$, and if $\mathscr{I}=\{X \subseteq \omega: \varphi(X) \neq$ $\omega\}$ is an ideal, then $\mathscr{I}$ is a nice ideal.

Proof. (9.6) holds with $\mathscr{G}_{n}=\left\{F \in[\omega]^{<\omega}: n \in \varphi(F)\right\} \quad(n \in \omega)$.

Lemma 9.5. Let $\mathscr{I}$ be a nice ideal on $\omega$ and let $G \in \mathscr{G}=\mathscr{P}(\omega) \backslash \mathscr{I}$. Suppose that $\alpha<\omega_{1}$ and that $\mathscr{S} \subseteq[\omega]^{<\omega} \quad \alpha$-blocks $G$. Then, for any partition $\mathscr{S}=$ $\mathscr{S}_{0} \cup \mathscr{S}_{1}$, there are $i<2$ and $G^{\prime} \subseteq G$ such that $G^{\prime} \in \mathscr{G}$ and $\mathscr{S}_{i} \alpha$-blocks $G^{\prime}$. Proof. By induction on $\alpha$. The case $\alpha=0$ is trivial, so assume $\alpha>0$. Since $\mathscr{S}_{0} \alpha$-blocks any finite set, we may also assume that $[G]^{<\omega} \subseteq \mathscr{F}$. Let $G_{0}=\{x \in G: \mathscr{S}-\{x\} \quad \beta$-blocks $G$ for some $\beta<\alpha\}$. Then $G_{0} \in \mathscr{G}$ since $G \backslash G_{0}$ is finite. For each $x \in G_{0}$ choose $\beta_{x}<\alpha$ so that $\mathscr{S}-\{x\} \quad \beta_{x}$-blocks $G$. Since $\mathscr{I}$ is nice, we may write $\mathscr{G}=\bigcap_{n<\omega} \overline{\mathscr{G}}_{n}$, where $\mathscr{G}_{n} \subseteq[\omega]^{<\omega} \quad(n<\omega)$. Pick $H_{0} \subseteq G_{0}$ so that $H_{0} \in \mathscr{G}_{0}$. Since $H_{0}$ is finite, it follows from (9.2) and $\left|H_{0}\right|$ applications of the induction hypothesis, that there is $G_{1} \subseteq G_{0}$ such that $G_{1} \in \mathscr{G}$ and

$$
\left(\forall x \in H_{0}\right) \quad\left(\mathscr{S}_{i(x)}-\{x\} \beta_{x} \text {-blocks } G_{1} \text { for some } i(x)<2\right) .
$$

Continuing in this manner we find sets $G_{n}, H_{n} \quad(n<\omega)$ so that $G_{n} \supseteq H_{n} \in \mathscr{G}_{n}$, $G_{n} \supseteq G_{n+1} \in \mathscr{G}$ and

$$
\left(\forall x \in H_{n}\right) \quad\left(\mathscr{S}_{i(x)}-\{x\} \beta_{x} \text {-blocks } G_{n+1} \text { for some } i(x)<2\right) .
$$

Let $H^{\prime}=\bigcup_{n<\omega} H_{n}$. Then $G \supseteq H^{\prime} \in \mathscr{G}$. Since $H^{\prime} \backslash G_{n}$ is finite for each $n<\omega$, it follows from (9.2) that $\mathscr{S}_{i(x)}-\{x\} \quad \beta_{x}$-blocks $H^{\prime}$ for each $x \in H^{\prime}$. Write $H^{\prime}=H^{(0)} \cup H^{(1)}$, where $H^{(i)}=\left\{x \in H^{\prime}: i(x)=i\right\}$. Then, for $i<2, H^{(i)} \subseteq G$ and $\mathscr{S}_{i} \alpha$-blocks $H^{(i)}$. Finally, since $\mathscr{I}$ is an ideal, there is some $i<2$ such that $H^{(i)}=G^{\prime} \in \mathscr{G}$.

We are now ready to prove Theorem 2.6, that an algebraic closure with no infinite independent set has a finite cardinal representation (i.e., $\mathbf{R}$ is true).

Proof of Theorem 2.6. By (9.5) it will suffice to prove that $\mathbf{P}(\alpha)$ is true for every $\alpha<\omega_{1}$. We do this by induction on $\alpha$. The case $\alpha=0$ is vacuous since $\varnothing \notin \operatorname{DEP}(\varphi)$. So assume that $0<\alpha<\omega_{1}$ and that $\mathbf{P}(\beta)$ holds for 
every $\beta<\alpha$. By Lemma 9.3 it is enough to prove $\mathbf{Q}(\alpha)$. So, assume that $\varphi$ is an algebraic closure on $\omega$, that $\mathscr{S}=\operatorname{DEP}(\varphi) \alpha$-blocks $\omega$, and that $\mathscr{I}=\{X \subseteq \omega: \varphi(X) \neq \omega\}$ is an ideal. We have to show that $\varphi$ has a finite cardinal representation or, by Theorem 2.8 , that $\varphi$ has a finite weak cardinal representation. If $\varphi(F)=\omega$ for some finite set $F$, then clearly $\varphi \in$ FCR and so we may assume that $[\omega]^{<\omega} \subseteq \mathscr{I}$.

Since $\mathscr{S} \alpha$-blocks $\omega$, there is a finite set $F \subseteq \omega$ such that, for each $n \in$ $\omega \backslash F$, there is an ordinal $\beta_{n}<\alpha$ such that $\mathscr{S}-\{n\} \quad \beta_{n}$-blocks $\omega$. Write $\mathscr{S}-\{n\}=\mathscr{S}_{n, 0} \cup \mathscr{S}_{n, 1}$, where $\mathscr{S}_{n, 0}=\{S \in \mathscr{S}-\{n\}: n \notin \varphi(S)\}, \mathscr{S}_{n, 1}=$ $\{S \in \mathscr{S}-\{n\}: n \in \varphi(S)\}$. Let $\mathscr{G}=\mathscr{P}(\omega) \backslash \mathscr{I}=\{X \subseteq \omega: \varphi(X)=\omega\}$.

Case 1. For some $n \in \omega \backslash F$, there is a set $H \in \mathscr{G}$ such that $\mathscr{S}_{n, 0} \beta_{n}$-blocks $H$.

Define an algebraic closure $\hat{\varphi}$ on $H$ by setting $\hat{\varphi}(X)=\varphi(X \cup\{n\}) \cap H$ $(\varnothing \neq X \subseteq H)$. Let $\widehat{\mathscr{S}}=\operatorname{DEP}(\hat{\varphi})=\left\{X \in[H]^{<\omega}: X\right.$ is $\hat{\varphi}$-dependent $\}$. If $X \in \mathscr{S}_{n, 0} \cap[H]^{<\omega}$, then $n \notin \varphi(X)$ and since $X \cup\{n\}$ is $\varphi$-dependent, there is $x \in X$ such that $x \in \varphi((X \cup\{n\}) \backslash\{x\}) \cap H$, i.e., $X \in \widehat{\mathscr{S}}$. Therefore, by (9.3), $\widehat{\mathscr{S}} \beta_{n}$-blocks $H$. It follows by the induction hypothesis, $\mathbf{P}\left(\beta_{n}\right)$, that $\hat{\varphi}$ has a finite cardinal representation, say $\mathscr{A}$. Then $\mathscr{A} \cup\{\{n\}\}$ is a finite weak cardinal representation of $\varphi$.

Case 2. For no $n \in \omega \backslash F$ is there a set $H \in \mathscr{G}$ such that $\mathscr{S}_{n, 0} \beta_{n}$-blocks $H$.

Since $\mathscr{I}$ is nice by Lemma 9.4, it follows from Lemma 9.5 that, for every $n \in \omega \backslash F$ and $G \in \mathscr{G}$, there is a set $H \subseteq G$ such that $H \in \mathscr{G}$ and $\mathscr{S}_{n, 1}$ $\beta_{n}$-blocks $H$; and so $\mathscr{S}_{n, 1}$ blocks $H$ by Lemma 9.2. Therefore, there are sets $H_{n} \in \mathscr{G} \quad(n<\omega)$ such that $H_{0} \supseteq H_{1} \supseteq \cdots$ and, for $n \in \omega \backslash F, \mathscr{S}_{n, 1}$ blocks $H_{n}$. Since each $H_{n}$ is infinite, there is an infinite set $A \subseteq \omega$ such that $\left|A \backslash H_{n}\right|<\omega$ for all $n<\omega$. It follows that $\mathscr{S}_{n, 1}$ blocks $A$ for every $n \in \omega \backslash F$ and so $\varphi(X) \supseteq \omega \backslash F$ for every $X \in[A]^{\omega}$. Thus $\{A, F\}$ is a weak cardinal representation for $\varphi$.

We conclude this section by proving the following self-strengthening of Theorem 2.6.

Theorem 9.6. Let $\varphi$ be a closure on $\omega$. If every infinite subset of $\omega$ contains a finite $\varphi$-dependent subset, then $\varphi$ has a finite cardinal representation.

Proof. For $X \subseteq \omega$ define $\varphi^{\prime}(X)=\bigcup\left\{\varphi(Y): Y \in[X]^{<\omega}\right\}$. Then $\varphi^{\prime}$ is an algebraic closure on $\omega$, and ind $\left(\varphi^{\prime}\right) \leq \omega$. By Theorem 2.6, $\varphi^{\prime}$ has a finite cardinal representation. Since $\varphi^{\prime}(X) \subseteq \varphi(X)$ for all $X \subseteq \omega$, any weak cardinal representation of $\varphi^{\prime}$ is also a weak cardinal representation of $\varphi$. Hence $\varphi$ has a finite weak cardinal representation and the result follows from Theorem 2.8 .

\section{Preclosures on $\omega$ with $\operatorname{ind}(\omega)<\omega$}

The key idea that we use for the proof of Theorem 2.7 is contained in the following lemma. 
Lemma 10.1. Let $\varphi$ be a preclosure on $\omega$ such that $\operatorname{dim}(\varphi)=\omega$. Then there is an infinite set $A \subseteq \omega$ such that (i) $A \cap \varphi(X)$ is finite for every finite set $X \subseteq \omega$, and (ii) if $y \in \omega, X \in[\omega]^{<\omega}, B \in[A]^{\omega}$, and $y \notin \varphi(X \cup B)$, then there is $a$ finite set $F \subseteq A$ such that $y \notin \varphi(X \cup(A \backslash F))$.

Proof. Choose $a_{n} \in \omega \backslash \varphi\left(\{i: i<n\} \cup\left\{a_{i}: i<n\right\}\right) \quad(n<\omega)$. Then any infinite subset, $A$, of the infinite set $A^{*}=\left\{a_{n}: n<\omega\right\}$ satisfies (i).

Let $\omega \times[\omega]^{<\omega}=\left\{\left(y_{n}, X_{n}\right): n<\omega\right\}$. Put $B_{0}=A^{*}$. Let $n<\omega$ and suppose that $B_{n} \in\left[A^{*}\right]^{\omega}$ has already been defined. If there is an infinite set $C \subseteq B_{n}$ such that $y_{n} \notin \varphi\left(X_{n} \cup C\right)$, then let $B_{n+1}$ be such a $C$. If there is no such $C$, put $B_{n+1}=B_{n}$. Then $B_{0} \supseteq B_{1} \supseteq \cdots$ and we choose $A \in\left[A^{*}\right]^{\omega}$ so that $\left|A \backslash B_{n}\right|<\omega$ for all $n<\omega$. We claim that (ii) holds. For if $(y, X) \in \omega \times[\omega]^{<\omega}$, $B \in[A]^{\omega}$, and $y \notin \varphi(X \cup B)$, then there is some $n<\omega$ such that $(y, X)=$ $\left(y_{n}, X_{n}\right)$ and then by the choice of $B_{n+1}$ we have $y \notin \varphi\left(X \cup B_{n+1}\right)$ and hence $y \notin \varphi(X \cup(A \backslash F))$ for some finite set $F$.

Proof of Theorem 2.7. Let $\varphi$ be a preclosure on $\omega$ with $\operatorname{ind}(\varphi)=k<\omega$. We will prove, by induction on $k$, that $\varphi$ has a cardinal representation, $\mathscr{A}$, of cardinality $|\mathscr{A}| \leq k$.

If $\varphi(X)=\omega$ for some finite set $X$ and $X$ is minimal, then $\{X\}$ is a cardinal representation of $\varphi$ (and $|\{X\}|=1 \leq k)$. Therefore, we may assume that $\varphi(X) \neq \omega$ for all $X \in[\omega]^{<\omega}$ and so there is a set $A \in[\omega]^{\omega}$ such that (i) and (ii) of Lemma 10.1 hold.

Let $E_{1}=\bigcap\left\{\varphi(B): B \in[A]^{\omega}\right\}$. If $E_{1}=\omega$ (as it is if $\left.k=1\right)$, then $\{A\}$ is a cardinal representation of $\varphi$. So we may assume that $E_{1} \neq \omega$ and that $k>1$. Now consider the preclosure $\varphi^{\prime}$ defined on $\omega$ by setting $\varphi^{\prime}(Y)=\bigcap\{\varphi(Y \cup B)$ : $\left.B \in[A]^{\omega}\right\}$ for $\varnothing \neq Y \subseteq \omega$. Suppose that $X \in[\omega]^{k}$ is $\varphi^{\prime}$-independent. Then $x \notin \varphi^{\prime}(X \backslash\{x\})$ for each $x \in X$ and so (since $\left.X \backslash\{x\} \neq \varnothing\right)$ there is $B_{x} \in[A]^{\omega}$ such that $x \notin \varphi\left((X \backslash\{x\}) \cup B_{x}\right)$. Therefore, by (ii), there is a finite set $F_{x} \subseteq A$ such that $x \notin \varphi\left((X \backslash\{x\}) \cup\left(A \backslash F_{x}\right)\right)$. By (i), $\varphi(X) \cap A$ is finite, and so the set $A_{1}=A \backslash\left(\varphi(X) \cup \bigcup\left\{F_{x}: x \in X\right\}\right)$ is infinite. But this is a contradiction since $\{y\} \cup X \in[\omega]^{k+1}$ and is $\varphi$-independent for $y \in A_{1}$. It follows that $\operatorname{ind}\left(\varphi^{\prime}\right)<k$ and so by the induction hypothesis $\varphi^{\prime}$ has a cardinal representation $\mathscr{A}^{\prime}$ with $\left|\mathscr{A}^{\prime}\right|<k$. The result now follows from Theorem 2.8 since $\mathscr{A}^{\prime} \cup\{A\}$ is a weak cardinal representation of $\varphi$.

By a similar argument to the one above we can also prove the following theorem.

Theorem 10.2. Let $n<\omega$ and suppose that $\varphi$ is a preclosure on $\omega$ such that $\operatorname{DEP}(\varphi)=\left\{X \in[\omega]^{<\omega}: X\right.$ is $\varphi$-dependent $\}$-blocks $\omega$. Then $\varphi$ has a cardinal representation $\{F\} \cup \mathscr{A}$, with $F$ finite and $|\mathscr{A}|<n$.

Note that, if $\varphi$ is a preclosure on $\omega$ such that $\operatorname{ind}(\varphi)=k<\omega$, then $\operatorname{DEP}(\varphi) \supseteq[\omega]^{k+1}$ and so $\operatorname{DEP}(\varphi)(k+1)$-blocks $\omega$. Therefore, by Theorem 10.2 , it follows that $\varphi$ has a cardinal representation of the form $\{F\} \cup \mathscr{A}$, with 
$F$ finite and $|\mathscr{A}| \leq k$. This is slightly weaker than the conclusion of Theorem 2.7, but the hypothesis of Theorem 10.2 is also weaker.

In order to prove Theorem 10.2, we first establish a simple lemma.

Lemma 10.3. Let $n<\omega ; \mathscr{S}, \mathscr{S}^{\prime} \subseteq[\omega]^{<\omega}$. If $\mathscr{S}(n+1)$-blocks $\omega, F \subseteq \omega$ is finite, and $\mathscr{S}^{\prime} \supseteq\left\{X \in[\omega]^{n}: \mathscr{S}-X\right.$ 1-blocks $\left.\omega\right\}$, then $\mathscr{S}^{\prime} n$-blocks $\omega$.

Proof. For $n=0$ this is clear. Let $n>0$ and use induction. For all but finitely many $x \in \omega$ we have that $\mathscr{S}-\{x\}$-blocks $\omega, \mathscr{S}^{\prime}-\{x\} \supseteq\{X \in$ $[\omega \backslash(F \cup\{x\})]^{n-1}:(\mathscr{S}-\{x\})-X$ 1-blocks $\left.\omega\right\}$ and so $\mathscr{S}^{\prime}-\{x\}$ (n-1)-blocks $\omega$. Hence $\mathscr{S}^{\prime} n$-blocks $\omega$.

Proof of Theorem 10.2. By Theorem 2.8 it will be enough to show that $\varphi$ has a weak cardinal representation of the kind described. Since a $\varphi$-dependent set contains at least two elements (since $\varphi(\varnothing)=\varnothing$ ), the cases $n=0$ and $n=1$ of the theorem are vacuous. We assume $n \geq 2$.

The result is obvious if $\varphi(X)=\omega$ for some finite set $X$. Thus we may assume that $\varphi(X) \neq \omega$ for $X \in[\omega]^{<\omega}$ and hence there is an infinite set $A \subseteq \omega$ such that (i) and (ii) of Lemma 10.1 hold.

If $n=2$, the hypothesis implies that there is a finite set $F$ such that $\operatorname{DEP}(\varphi)-\{x\}$ 1-blocks $\omega$ for all $x \in \omega \backslash F$. Therefore, for each $x \in \omega \backslash F$, there is a finite set $F_{x}$ such that $\{x, y\} \in \operatorname{DEP}(\varphi)$ for every $y \in \omega \backslash F_{x}$. Let $B \in[A]^{\omega}$. If $x \in \omega \backslash F$, there is some $y \in B \backslash\left(F_{x} \cup \varphi(\{x\})\right)$, and so $x \in \varphi(\{y\})$. This shows that $\varphi(B) \supseteq \omega \backslash F$. It follows that $\{F, A\}$ is a weak cardinal representation of $\varphi$ as required. Now assume that $n>2$ and use induction on $n$.

As in the proof of Theorem 2.7, we define a closure $\varphi^{\prime}$ on $\omega$ by setting $\varphi^{\prime}(Y)=\bigcap\left\{\varphi(Y \cup B): B \in[A]^{\omega}\right\}$ for $\varnothing \neq Y \subseteq \omega$. We claim that $\operatorname{DEP}\left(\varphi^{\prime}\right)$ $(n-1)$-blocks $\omega$. By Lemma 10.3, to prove the claim it will be enough to show that $\operatorname{DEP}\left(\varphi^{\prime}\right) \supseteq\left\{X \in[\omega]^{n-1}: \operatorname{DEP}(\varphi)-X\right.$ 1-blocks $\left.\omega\right\}$. Suppose $X \in[\omega]^{n-1}$ and that $\operatorname{DEP}(\varphi)-X$ 1-blocks $\omega$. Then, for all but finitely many $y \in \omega$, $\{y\} \in \operatorname{DEP}(\varphi)-X$, so that $X \cup\{y\}$ is $\varphi$-dependent. If $X$ is $\varphi^{\prime}$-independent, then by the same argument that was used in the proof of Theorem 2.7, it follows that $X \cup\{y\}$ is $\varphi$-independent for all but finitely many elements $y \in A$. But this is a contradiction, and so $X \in \operatorname{DEP}\left(\varphi^{\prime}\right)$ and the claim is proved.

By the induction hypothesis $\varphi^{\prime}$ has a cardinal representation of the form $\mathscr{A}_{1}=\{F\} \cup \mathscr{B}$, with $|\mathscr{B}|<n-1$. Then $\mathscr{A}=\mathscr{A}_{1} \cup\{A\}$ is a weak cardinal representation of $\varphi$.

\section{Negative Results}

Theorem 11.1 shows that the condition that $\varphi$ be algebraic is needed for Theorem 2.6. It also justifies our earlier remark that nothing can be said about the size of the cardinal representation of a closure, $\varphi$, on $\omega$ with $\operatorname{ind}(\varphi) \leq \omega$ whose existence is guaranteed by Theorem 2.5 . 
Theorem 11.1. There is a $T_{1}$-topology on $\omega$ such that, denoting by $\varphi(X)$ the closure of $X$ in this topology, we have:

(i) $\varphi$ has no infinite independent set;

(ii) $\varphi$ has no countable cardinal representation (and hence no finite weak cardinal representation);

(iii) it is consistent that $2^{\aleph_{0}}$ is arbitrarily large and that $\varphi$ has no cardinal representation of size less than $2^{\aleph_{0}}$.

Proof. Let $\mathscr{S} \subseteq[\omega]^{\omega}$ be an infinite maximal almost disjoint (mad) family (i.e., $|A \cap B|<\omega$ for $A, B \in \mathscr{S}$ and $A \neq B$; and whenever $X \in[\omega]^{\omega}$, $X \cap S$ is infinite for some $S \in \mathscr{S})$. Let $\mathscr{K}=\{\omega\} \cup\left\{F \cup \bigcup \mathscr{F}: F \in[\omega]^{<\omega}\right.$, $\left.\mathscr{F} \in[\mathscr{S}]^{<\omega}\right\}$. Note that $[\omega]^{<\omega} \subseteq \mathscr{K}, \omega \in \mathscr{K}$, and $\mathscr{K}$ is closed under finite unions and arbitrary intersections. Thus $\mathscr{K}$ is the family of all closed subsets for a $T_{1}$-topology on $\omega$. Let $\varphi(X)=\bigcap\{K: X \subseteq K \in \mathscr{K}\} \quad(X \subseteq \omega)$. If $X$ is any infinite subset of $\omega$, then there is $S \in \mathscr{S}$ such that $|X \cap S|=\omega$ and, for $x \in X \cap S$, we have $\varphi(X \cap S \backslash\{x\})=S$. Thus, there is no infinite $\varphi$-independent set (and so the topology is not $T_{2}$ since there is no infinite discrete subspace).

To prove (ii) we show that, for any countable set $\mathscr{A} \subseteq \mathscr{P}(\omega)$, either (a) there is $X \subseteq \bigcup \mathscr{A}$ such that $\varphi(X) \neq \omega$ and $|X \cap A|=|A|(\forall A \in \mathscr{A})$, or (b) $\varphi(\bigcup \mathscr{A} \backslash A)=\omega$ for some $A \in \mathscr{A}$. Let $\mathscr{S}^{\prime}=\{S \in \mathscr{S}:|S \cap \bigcup \mathscr{A}|=$ $\omega\}$. Then $\left|\mathscr{S}^{\prime}\right| \neq \omega$ by the assumed maximality of $\mathscr{S}$. If $\left|\mathscr{S}^{\prime}\right|<\omega$, then $F=\bigcup \mathscr{A} \backslash \bigcup \mathscr{S}^{\prime}$ is finite, and (a) holds with $X=\bigcup \mathscr{A}$ since $\varphi(\bigcup \mathscr{A})=$ $F \cup \cup \mathscr{S}^{\prime} \neq \omega$. Therefore, we may assume that $\left|\mathscr{S}^{\prime}\right|>\omega$. If, for some $S \in \mathscr{S},|A \cap S|=|A|$ for every $A \in \mathscr{A}$, then $\varphi(S \cap \bigcup \mathscr{A})=S \neq \omega$ and again (a) holds. Therefore, for each $S \in \mathscr{S}$, we may assume that there is some $A(S) \in \mathscr{A}$ such that $S \cap A(S)$ is finite. There is $\mathscr{S}^{\prime \prime} \in\left[\mathscr{S}^{\prime}\right]^{\omega}$ such that $A(S)=A$ for every $S \in \mathscr{S}^{\prime \prime}$. Since $S \cap(\bigcup \mathscr{A} \backslash A)$ is infinite for every $S \in \mathscr{S}^{\prime \prime}$, it follows that $\varphi(\bigcup \mathscr{A} \backslash A)=\omega$ and (b) holds.

The proof of (iii) is similar. We use the known fact (K. R. Milliken, Theorem 2.2 of [7]), that the following statements $\mathscr{M}_{1}$ and $\mathscr{M}_{2}$ are equivalent, and that $\mathscr{M}_{2}$ is consistent with an arbitrarily large choice for $2^{\aleph_{0}}$.

$\mathscr{M}_{1}$ : there is an infinite mad family $\mathscr{S} \subseteq[\omega]^{\omega}$ such that for each $X \in[\omega]^{\omega}$, EITHER (i) $X \subseteq \cup \mathscr{S}^{\prime}$ for some $\mathscr{S}^{\prime} \in[\mathscr{S}]^{<\omega}$, OR (ii) $|\{S \cap X: S \in \mathscr{S}\}|=2^{\mathrm{\aleph}_{0}}$.

$\mathscr{M}_{2}$ : There is an infinite mad family $\mathscr{S} \subseteq[\omega]^{\omega}$ such that, for each $X \in[\omega]^{\omega}$, EITHER (a) $X \subseteq \bigcup \mathscr{S}^{\prime}$ for some $\mathscr{S}^{\prime} \in[\mathscr{S}]^{<\omega}$, OR (b) $S \subseteq X$ for some $S \in \mathscr{S}$.

Thus we may assume that the mad family $\mathscr{S}$ satisfies $\mathscr{M}_{1}$. In this case, if $\mathscr{A} \subseteq \mathscr{P}(\omega)$ and $|\mathscr{A}|<2^{\aleph_{0}}$, and if $\mathscr{S}^{\prime}$ is defined as in the proof of (ii), then either $\mathscr{S}^{\prime}$ is finite or $\left|\mathscr{S}^{\prime}\right|=2^{\aleph_{0}}$. The argument continues exactly as in the proof of (ii).

Our next example (Theorem 11.2) shows that the condition that $\varphi$ have no infinite independent set is essential for Theorems 2.5 and 2.6. 
Theorem 11.2. There is a closure of character 3 on $\omega$ (which has infinite independent sets) which has no cardinal representation (and therefore has no finite weak cardinal representation).

Proof. Let $P_{n}=\{2 n, 2 n+1\} \quad(n<\omega)$. For $X \subseteq \omega$, define $\varphi(X)=X \cup$ $\bigcup\left\{P_{n}: P_{n} \cap X \neq \varnothing\right.$ and $P_{m} \subseteq X$ for some $\left.m>n\right\}$. Then $\varphi$ is a closure of character 3. Suppose for contradiction that $\mathscr{A}$ is a cardinal representation for $\varphi$. Then $\bigcup \mathscr{A} \cap P_{n} \neq \varnothing$ for every $n<\omega$ and $P_{n} \subseteq \bigcup \mathscr{A}$ for infinitely many $n$. Choose $n<\omega$ so that $P_{n} \subseteq \bigcup \mathscr{A}$. Since $\varphi\left(\bigcup \mathscr{A} \backslash P_{n}\right) \neq \omega$, it follows that $\left|A \cap\left(\bigcup \mathscr{A} \backslash P_{n}\right)\right|<|A|$ for some $A \in \mathscr{A}$. Therefore, $A$ is finite and there is $x \in P_{n} \cap A \neq \varnothing$. Now $\varphi(\bigcup \mathscr{A} \backslash\{x\})=\omega$, but $|(\bigcup \mathscr{A} \backslash\{x\}) \cap A|<|A|$, and this contradicts the assumption that $\mathscr{A}$ is a cardinal representation.

As we already remarked (Problem 2), we do not know if there is a closure of character two which has no cardinal representation.

\section{REFERENCES}

1. G. Birkhoff, Lattice theory, 3rd ed., Amer. Math. Soc. Colloq. Publ., Amer. Math. Soc., Providence, R.I., 1967.

2. P. M. Cohn, Universal algebra, Reidel, Dordrecht, 1981.

3. R. P. Dilworth, A decomposition theorem for partially ordered sets, Ann. of Math. (2) 51 (1950), 161-166.

4. D. Duffus and M. Pouzet, Representing ordered sets by chains, Ann. Discrete Math. 23 (1984), 81-98.

5. P. Erdös and A. Tarski, On families of mutually exclusive sets, Ann. of Math. (2) 44 (1943), 315-329.

6. A. Hajnal, Proof of a conjecture of S. Ruziewicz, Fund. Math. 50 (1961), 123-128.

7. Keith R. Milliken, Completely separable families and Ramsey's theorem, J. Combin. Theory Ser. A 19 (1975), 318-334.

8. E. C. Milner and M. Pouzet, On the cofinality of partially ordered sets, Ordered Sets (Banff, 1981) (I. Rival, ed.), Reidel, 1982, pp. 279-298.

9. __ On the independent subsets of a closure system with singular dimension, Algebra Universalis 21 (1985), 25-32.

10. Z. Nagy and Z. Szentmiklóssy, On the representation of tournaments, preprint.

11. S. Todorčević, Directed sets and cofinal types, Trans. Amer. Math. Soc. 290 (1985), 711-723.

Department of Mathematics, University of Kansas, Lawrence, Kansas 66045

Department of Mathematics, University of Calgary, Calgary, Alberta, Canada T2N $1 \mathrm{~N} 4$

Département de MathématiQues, Université Claude Bernard, Lyon 1, France 